University of Nebraska - Lincoln

DigitalCommons@University of Nebraska - Lincoln

Robert Katz Publications

Research Papers in Physics and Astronomy

January 1993

\title{
A Model of Cell Damage in Space Flight
}

Robert Katz

University of Nebraska-Lincoln, rkatz2@unl.edu

F. A. Cucinotta

NASA Langley Research Center, Hampton, Virginia, francis.cucinotta@unlv.edu

J. W. Wilson

NASA Langley Research Center, Hampton, Virginia

J. L. Shinn

NASA Langley Research Center, Hampton, Virginia

Duc M. Ngo

Old Dominion University, Norfolk, Virginia

Follow this and additional works at: https://digitalcommons.unl.edu/physicskatz

Part of the Physics Commons

Katz, Robert; Cucinotta, F. A.; Wilson, J. W.; Shinn, J. L.; and Ngo, Duc M., "A Model of Cell Damage in Space Flight" (1993). Robert Katz Publications. 80.

https://digitalcommons.unl.edu/physicskatz/80

This Article is brought to you for free and open access by the Research Papers in Physics and Astronomy at DigitalCommons@University of Nebraska - Lincoln. It has been accepted for inclusion in Robert Katz Publications by an authorized administrator of DigitalCommons@University of Nebraska - Lincoln. 


\title{
A MODEL OF CELL DAMAGE IN SPACE FLIGHT
}

\author{
Robert Katz \\ University of Nebraska \\ Lincoln, Nebraska 68588-0150
}

F. A. Cucinotta, J. W. Wilson, and J. L. Shinn

NASA Langley Research Center

Hampton, Virginia 23665-5225

Duc M. Ngo

Department of Physics

Old Dominion University

Norfolk, Virginia 23508

\begin{abstract}
Cell damage by high LET radiations has been described by a phenomenological model (track theory) for 20 years and more. Molecules of biological significance (dry enzymes and viruses) act as 1 hit detectors. Recent additions to the class of 1-hit detectors are E. Coli B, and the creation of both single and double strand breaks in SV-40 virus in EO buffer, where indirect effects predominate. The response of cells (survival, transformation, chromosome aberration) to these radiations is typically described by a 4-parameter model whose numerical values are determined by fitting the equations of the theory to experimental data at high dose (typically above $1 \mathrm{~Gy}$ ) with bombardments with $\gamma$ rays and HZE particle beams, of the widest possible dynamic range. Once these parameters are determined the model predicts cellular response in any radiation environment whose particle-energy spectrum is known. Perhaps the central importance of the present model is the ability to estimate the response of a complex environment with many components from a limited set of laboratory data. For example, we have calculated cell survival after neutron irradiation, with mixtures of neutrons and $\gamma$ rays; cell survival and transformation after irradiation with HZE ions of different energies. The model does not yet include cellular repair. Although some hopeful approaches to repair dependence are now being developed. It does not include cancer induction, for the available data neither give the number of cells at risk or the number of cancers induced, and are thus not suited to our formulation.
\end{abstract}

Most recently NASA-Langley models of HZE beams, including projectile and target fragmentation, have been joined with the biological model. This combination has been tested against ground based radiobiological data for cell survival after irradiation with protons and HZE beams with good success. Where our earlier model failed downstream of the Bragg 
peak (for both protons and heavy ions) for want of a proper description of fragmentation the NASA-Langley model succeeds.

Based on this experimental validation of our procedures, we have initiated calculations of cellular damage in space flight from solar protons and galactic cosmic rays. Here we incorporate NASA models of cosmic rays, beam penetration, projectile and target fragmentation with track theory. The essential radiobiological theme is that knowledge of parameters extracted at high doses makes it possible for us to calculate the response of cells at the lowest possible doses of HZE particles when only intra track (ion-kill) effects are involved for which repair is known to be minimal. Our procedures here too have ground based experimental validation in recent work of Bettega et al. where measurements made of $\mathrm{RBE}$ with protons and alphas of the survival of $\mathrm{C} 3 \mathrm{H} 10 \mathrm{~T} \mathrm{~T}_{2} / 2$ cells, at doses down to $0.01 \mathrm{~Gy}$ are consistent with our predictions based on survival measurements made at high doses with $\gamma$ rays and HZE ions.

\section{INTRODUCTION}

Detectors of radiation differ according to whether single particle response is normally observed, as with nuclear emulsions, solid state nuclear track detectors, and scintillation counters, or whether the response is to beams of particles or photons in a gross macroscopic irradiation, as in radiobiology or in the alteration of bulk material properties by radiation. In the former case it is more natural to think in terms of track structure, while in the latter case one frequently refers to macroscopic dose (Katz, 1978). Response is then correlated to the physical description of these stimula. It is common to relate response to energy deposition (dose). Problems arise because response depends not only on total energy deposition but on the microscopic structure of that deposition and also on its time development. One analysis of these details is called microdosimetry, a subject that has stimulated many investigations. An alternate procedure favored here relates the observed effect to track structure for individual particles, which then may be related to macroscopic dose for gross irradiations. These perspectives are principally reported in the several Symposia on Microdosimetry sponsored by the Commission of European Communities.

The galactic cosmic ray (GCR) environment is the most complicated mixture of radiation components known. It is doubtful that the GCR will ever be adequately simulated in the laboratory for biological experiments. The primary role of track structure models will be to extrapolate laboratory response data to the GCR environment for the estimate of risk to biological tissues in space exposure. This is, we believe, to be a more practical approach to the issue of additivity of response of disparate components than the usual quality factor approach based on relative biological effectiveness (RBE) which has been used with limited success in terrestrial radiation protection. We will now discuss the current approaches to the question of additivity being pursued by various groups.

\section{ENERGY DEPOSITION IN SMALL VOLUMES: MICRODOSIMETRY}

One way to analyze the stimulus to biological systems is to examine the details of energy deposition in small volumes, sized to represent what are thought to be critical targets within the cell. Experimentally small gaseous proportional counters are used whose diameter, scaled to the density of tissue, is from micrometers to nanometers in unit density material. The critical targets are then considered to be either the nucleus of a mammalian cell or a chromosome, or a small region of DNA. The fluctuations of the energy deposited within the small target region is assumed related to biological response. A Monte Carlo simulation of a radiation field can yield a similar decomposition.

Even when one has a complete microdosimetric description of the radiation environment, the problem remains as to how that may be interpreted to predict the response of a detector. As yet we have no means of calibrating response in terms of the statistical distribution of energy depositions in small volumes. Nor do we know what volume is appropriate. It is on this level that microdosimetry has not been able to make extensive quantitative predictions, 
nor is it able to yield calculations of cross section. But the study has yielded many interesting insights into the structure of a radiation field (Goodhead, 1988). The small counter has found application in monitoring neutron beams used in radiotherapy and in other radiation fields including space and high altitude aircraft. Most instruments are used in practice to derive averages over quality factors discussed in section 5 .

\section{CROSS SECTION}

A second approach has been to attempt to mimic the kind of logical structure used in experiments in physics; that is, to describe the relevant interactions through the concept of an interaction cross section. We imagine that a projectile passing down a channel $1 \mathrm{~cm}^{2}$ in area interacts with a target located somewhere within that channel, and measure the fraction of successes after a large number of identical repeated trials. This probability is represented as though it is a geometrical target, as the cross section $\sigma$ in $\mathrm{cm}^{2}$ to the cross sectional area of the channel. We then speak of the action cross section even if the observed end point is achieved as a result of many internal changes stimulated by the initial interaction. Here we make no attempt to examine the internal processes mechanistically. The target is a black box. We know only the incident radiation and the observed end point. In radiobiology the concept of cross section is sometimes used in ways which depart from its original physical meaning. This can lead to misinterpretations of experimental data (Katz, 1990). Curtis et al. (1990) has recommended an additivity formalism based on a limited set of data for Harderian gland tumorgenesis using a cross section like formalism as an alternative to the use of RBE.

\section{G VALUE}

When a projectile impinges on a thin slice of matter containing $N$ targets $/ \mathrm{cm}^{3}$, the number of observable events per $\mathrm{cm}$ of path length is $n=\sigma N$. If the energy deposited per $\mathrm{cm}$ of path length is $L$ (LET = Linear Energy Transfer, or stopping power), the number of observed events per unit of energy deposited, the $G$ value is $G=n / L=\sigma N / L$. The cross section is a function of the medium, the end point, and the character of the projectile: if a photon its energy, if a naked charged particle its charge and speed, if a nucleus partially clothed with electrons its effective charge and speed. This formulation of the $G$ value has been used in the analysis of heavy ions radiolysis (Katz and Huang, 1989). In dealing with liquids where the meaning of $N$ may be obscure we have calculated the $G$ value for heavy ion bombardment from calculated values of the RBE and known $G$ values for $\gamma$ irradiation, as in the Fricke dosimeter (Katz, Sinclair and Waligorski, 1986). In other cases we have tried to relate $N$ to a fitted target size. Nuclear collisions are here neglected except as a source of charged fragments.

If the $G$ value is normalized to molecular weight, expressed as events/rad/Dalton rather than events $/ 100 \mathrm{eV}$, we find it proportional to the RBE for dry 1-hit detectors for which the "target molecular weight" is equal to the true molecular weight (Katz, 1990).

\section{RELATIVE EFFECTIVENESS; QUALITY FACTOR}

When intense neutron environments became available, the existing body of biological response data was mainly for X-ray and $\gamma$-ray sources. The first efforts at protection attempted to scale the known $\mathrm{X}$-ray and $\gamma$-ray risks according to equivalent neutron dose giving rise to the concepts of radiation quality and RBE. For the case of space radiations, we should like to relate the response of our detectors to energetic heavy ions, that is to high LET radiations, to their response to photons and electrons, that is to low LET radiations. In radiobiology the ratio of the dose of gamma rays to that of another radiation which produced the same observed end point is called the relative biological effectiveness, the RBE. In radiotherapy this quantity is frequently taken to be a property of the two radiation fields, but it depends on the dose level, the dose rate and the end point as well. An extension of this idea used in radiation protection is called the quality factor estimated as the upper limit of RBE values 
for a selected set of biological endpoints judged relevant to human risk and taken solely as a function of LET. An important unresolved question is whether an upper limit or maximum $\mathrm{RBE}$ is achieved at the low exposures of interest for radiation protection. The quality factor is used to convert a measured dose in Gray into an effective dose reported (not measured) in Sieverts. We note that the Sievert is not a directly measurable quantity and thus violates the underlying philosophy through which physical units are defined. The redeeming quality of the unit is that risk estimates based on Sieverts should be conservative providing the quality factor is adequately defined. The conservative nature of the method may also be an unacceptable burden in many operations, especially in space.

\section{RADIATION QUALITY}

An irradiation with photons leads to secondary electrons randomly dispersed through a medium. The initial energy spectrum of these electrons and their path lengths depends on the initial energy spectrum of the photons. An irradiation with a beam of heavy ions yields a random distribution of heavy ion paths, with the secondary electrons (delta rays) clustered around each ion's path (correlation effects), and having a different energy distribution. Hence the $\delta$ rays are not truly randomly distributed. They are clustered about the paths of heavy ions. This leads to a basic difference in the manner in which their effects are approached statistically (spatial correlations). In the present model we speak of "gamma-kill" to describe the effects of a random distribution of spatially uncorrelated electrons, and of "ion-kill" to describe the effects of the spatial correlations within single particle tracks. At high fluences of low LET ions, where only a fraction of the intersected targets is inactivated, we approximate the effect of the sparsely distributed and overlapping $\delta$ rays from several ions as due to randomly distributed electrons, and so we speak of gamma-kill as responsible for part of the effect from beams of some heavy ions.

With photons minutes may elapse for the traversal of secondary electrons from different photons through a target (uncorrelated temporal events). With heavy ions a single ion and its delta rays pass through a target in an extremely short time for the projectile moves at nearly the speed of light through a target whose diameter is of order 1 micron. These differences lead to a variation in response to radiations of different admixtures of photons and heavy ions at the same dose. When the temporal correlation time is on the order of the cell repair time then response also varies with dose rate or fractionation schedule. The dependence on dose rate or fraction schedule is due in part to radiation quality. The quality factor taken as the low dose rate limit RBE's in an attempt to normalize the biological effects of radiations of different quality. This assumes that it is logically correct to represent the response of a detector as a product of two separate factors, one of which is the dose while the other is the quality.

\section{RADIAL DOSE DISTRIBUTION}

For purposes of track structure calculations we need the radial distribution of dose, from delta rays and the primary interactions, about the path of an energetic charged particle (Waligorski, Hamm and Katz, 1986). We presently use an analytic representation of the results of a Monte Carlo calculation made for liquid water for this purpose. More recently we have extended this model to include some solids used as radiation detectors (Katz et al., 1990). Additional information about both theoretical and experimental determinations of the radial dose distribution may be found in a recent work by Katz and Varma (1990). We use this information in connection with the response of the detector to $\gamma$ rays to find the radial distribution of effect around a particle's path. Since we interpret the response to be the probability for activating a target, we can make a map of the radial distribution of activated targets. If we are interested in the opacity of a track in nuclear emulsion we can calculate the attenuation of a beam of light in a microscope photometer, as in the study of cosmic ray tracks (Katz and Kobetich, 1969). Alternatively we can integrate the probability radially to yield the cross section (Waligorski, Loh and Katz, 1987) for the interaction of a single ion with the target (Katz, 1978). 


\section{HITTEDNESS}

In radiobiology we are not yet able to measure the effect produced by the interaction of a single ion. Yet this is of central importance in estimating the effects of galactic cosmic rays in space flight. For this one needs to know the cross section. In track theory we wish to know whether a single particle, be it electron, proton, alpha particle, or whatever, is capable of inducing the tested end point with observable probability. We characterize these interactions through the concept of hittedness, borrowed from biological target theory (Dertinger and Jung, 1970). For a specific irradiation the appropriate hittedness is either the number of interactions between charged particles and target needed to induce the end point, or the number of incident particles which must bombard the target, whichever is smaller. If either a single particle or a single interaction leads to the event we will observe exponential response, as demanded by the cumulative Poisson distribution. If two electrons are required we expect to observe a response described by the 2-or-more hit cumulative Poisson distribution. But the inactivation may take place through the transit of a single $\alpha$ particle. In that case we expect to observe that the response to $\alpha$ particles is 1-or-more hit. We characterize the hittedness of a detector by its response to electrons or to gamma-rays. Experimentally if the response to the dose of gamma rays is exponential we speak of a 1-ormore hit detector.

\section{THE 1-HIT DETECTOR}

Most commonly radiation detectors can be described as 1-or-more hit detectors. We imagine the detector to be a collection of targets, sometimes explicit as in photographic emulsion, and sometimes implicit, as in a Fricke dosimeter, or in alanine. Each of these targets is capable of responding to the transit of a single electron of appropriate energy. The response is exponential; that is, it is linear at low dose and sublinear at high dose as the available targets tend to have been (in)activated. We speak of saturation or overkill at high dose. For 1-hit detectors the response to heavy ions is also exponential with dose or fluence.

To calculate the (in)activation cross section for a 1-hit detector we first find $P(D)$, the probability for target inactivation as a function of the dose $D$ of $\gamma$ rays. Next we fold this into the average radial dose distribution about an ions path, to find the probability for target inactivation $P(t)$ at radial distance $t$. We integrate $P(t)$ radially to find the cross section $\sigma$. When targets out to about 3 target diameters are all (in)activated we simply use the point distribution of dose in our calculations. If we must take into account effects closer than 3 target radii from the ion's path it is necessary to average the dose in the extended targets to accommodate the dose gradient, for the radial dose falls off essentially inversely as the square of the radial distance, out to a limit determined by the maximum $\delta$ ray penetration. This limiting distance, determined essentially by the speed of the ion, places an upper limit on the cross-section, observed experimentally in experiments with very heavy ions as "thin-down", so called because of the appearance of the tracks of heavy ions in electron sensitive emuisions, where the stopping end of a track looks like a sharpened pencil.

Typically for these detectors the cross section increases with an increase in $\left(Z^{*} / \beta\right)^{2}$ to a maximum (typically unrelated to target size) and then declines in thin-down.

The relative effectiveness defined biologically as RBE is equal to $\sigma E_{o} / L$, where $E_{o}$ is the $1 / \mathrm{e}$ dose or the dose for $37 \%$ survival. For 1 -hit detectors the RBE never exceeds 1 . The magnitude of the cross section is approximately determined by the radial distance at which the dose equals $E_{o}$. One may speak of the cross section as approximating the size of the damaged region, but it is inappropriate to speak of track size without specifying the end point. It is easy to estimate the cross section of a heavy ion with the grains of a nuclear emulsion from a microphotograph by estimating the radial distance at which about $63 \%$ of the grains are developed. There we note that for insensitive emulsions where the track resembles a string of beads, that the cross section is less than the grain size, while for a sensitive emulsion where the track resembles a hairy rope, that the cross section may be orders of magnitude greater than the grain size. 
Our first venture into the 1-hit detector was made for dry enzymes and viruses (Butts and Katz, 1968). This was shortly followed by a model for the response of nuclear emulsions (Katz and Kobetich, 1969), scintillation counters (Katz and Kobetich, 1968), TLD's, and subsequently of alanine (Waligorski et al., 1989), and most recently for E. Coli B (Katz and Zachariah, 1991). There are indications that CR-39, used as an etchable track detector is also a 1-hit detector (Katz, 1984).

The global applicability of the model of the 1-hit detector to a wide variety of detectors whose mechanisms are vastly different from each other is at first thought rather astonishing. It arises simply from the fact that in each case the end point is stimulated by the passage of a single electron through the target volume.

\section{SUPRALINEARITY; THE LINEAR QUADRATIC "MODEL"}

If a system has both 1 hit and 2 hit targets having different radiosensitivities and populations, we must expect that response will be linear at low dose, quadratic at intermediate doses, and finally saturating at high dose. We call such a response supralinear and have proposed such a model to explain supralinearity in TLD-100. Note that the concept of a 2-hit target requires only that two incident electrons are required to stimulate the end point. The response may arise after processing as well as in the initial interactions, for the present model treats each detector as a black box. But if there are not two varieties of response we cannot understand supralinearity in this model. Nor can we understand how a detector whose response to $\gamma$ rays is exponential can exhibit an RBE greater than 1 with heavy ions unless there are temporal effects hinging on the time difference between $\gamma$-ray and $\delta$ ray exposures. Such a time difference between hits appears explicitly in a kinetics model which may provide an approach to temporal effects.

In the same way we do not understand the basis of the linear quadratic formula widely used to fit radiobiological data, if there are not two types of targets within a cell. We note that the formula is simply the first two terms of a series expansion and is usually applied to data of very limited dynamic range. Further we note that there is no theory which can predict the values of either the linear or quadratic terms reliably, for radiations of different quality. Nevertheless these terms are liberally interpreted on the basis of such phrases as "could be" or "might be" though with equal validity one might insert "not".

Those who prefer to interpret data on the basis of hypothetical mechanisms (whose details are rarely accessible to experiment) object to our parametric formulations. Yet the model and its experimental parameters should not be dismissed lightly, for they may suggest mechanistic interpretations which supersede those presently popular (Goodhead, 1989). We need to be reminded that Newton's laws were stimulated by Kepler's phenomenology, and that quantum theory was stimulated by Planck's exercise in curve fitting.

\section{THE CELL SURVIVAL MODEL}

Biological cells require special consideration. For other detectors we assume, as in the case of nuclear emulsions, that there is a characteristic target size without internal structure. These are then characterized by the parameters $E_{o}$, the dose of $\gamma$ rays at which there is an average of 1 hit per target, $a_{D}$, the target radius, and $C_{3}$ the hittedness. We sometimes introduce a dimensionless parameter $\kappa$ proportional to $E_{o} a_{o}^{2}$. Biological cells have internal targets. We imagine the cells to resemble a bean bag in which the cell nucleus is the bag and the targets are the beans. We take it that the beans are 1-hit in character but that $m$ of the beans must be (in)activated to generate the observed response. We also imagine that there are beans well distributed through the bag so that an energetic ion passing through the bag has the possibility for inactivating $m$ beans. Such a model makes it possible to understand why flatted cells respond differently to $\alpha$ particles than rounded ones.

In this model the observed cross section is related to the size of the bean bag, the cell nucleus, while the variation of response with LET is related to the properties of the bean. To 
set up a model of cellular response we calculate the cross section for a hypothetical cluster of $m$ overlapping beans (Katz, Sharma, and Homagyoonfar, 1972) and then assume that the cross section for the bean bag is proportional to that of the cluster. Since our model is based on the radial distribution of dose from $\delta$ rays it automatically predicts thindown. For mammalian cells the fitted value of the $\kappa$ parameter suggests that the bean radius is about 1 micron, hinting that the target for cell killing may be a chromosome.

\section{THE MATHEMATICAL MODEL}

Detailed descriptions of the cellular track model have been given elsewhere. Here we present only the main concepts of the model and list the equations used in our calculations. Following our earlier studies of the appearance of particle tracks in nuclear emulsion (Katz and Kobetich, 1969), the model distinguishes between the "grain-count" regime where inactivations occur randomly along the particle's path, and the "track width" regime where the inactivations are distributed like a "hairy rope". The transition from the grain-count to track width regime trakes place in the neighborhood of $Z^{* 2} / \kappa \beta^{2}$ of about 4 ; at lower values we are in the graincount regime, at higher values in the track width regime. The quality $\kappa$ is a parameter of the model which combines both the target size and the characteristic dose of gamma rays at which there is an average of one hit per target. As in nuclear emulsions we speak of a thin down regime where the cross section is limited by the kinematic constraint on $\delta$ ray energies, but has nothing to do with the Bragg peak in stopping power nor with the changing effective charge of a slowing down ion.

To accommodate for the capacity of cells to accumulate sublethal damage, two modes of inactivation are identified, namely "ion-kill" (or "intratrack") and "gamma-kill" (or "intertrack"). In these two inactivation modes it is the statistical character of the inactivation which is changing rather than the fundamental physical interaction. Effects are referred to dose rather than to the number of electrons passing through the nucleus. We do not find justification for considering the stopping end of an electron track as a source of ion kill nor for the radial separation of a heavy ion track into core producing ion kill and penumbra producing gamma kill.

The model leads to the use of $Z^{* 2} / \beta^{2}$ as a plotting parameter superior to LET, now in wide use. At the stopping end of a track, at highest LET even this parameter fails for in the thin down regime the cross section depends on $\beta$, the relative speed of the ion. Here the cross section is sometimes plotted against the energy per unit mass, a related parameter. The model provides a basis for the meaning of low LET, based on the comparison of $Z^{* 2} / \beta^{2}$ with $\kappa$. Similarly low dose means low compared to $E_{o}$. The model explains why plots of extrapolated cross section (from the tail of a survival curve) tend to be single valued functions of LET at low LET (because the response is dominated by gamma kill) and why they are multiple valued (with $Z$ ) at high LET (because the response is dominated by ion kill and thin down). It explains why plots of RBE vs LET for biological cells pass through a maximum (when about half the intersected cells are killed in ion kill). It predicts that the RBE for lighter ions will be greater than the RBE for heavy ions at the same LET and the same survival level. This is because of the structure of particle tracks. At the same LET the heavier ions move faster. Its $\delta$ rays are fewer but more energetic. Gamma-kill is more likely, reducing the RBE.

\section{GAMMA KILL}

Cells not inactivated in the ion-kill mode can be sublethally damaged by the $\delta$-rays from the passing particle and then inactivated, in the gamma-kill mode, by cumulative addition of sublethal damage due to $\delta$ rays from other passing ions. Survival in the gamma-kill mode is taken to follow the $m$-target statistics of inactivation by secondary electrons from X-ray or gamma-ray photons. 


\section{MATHEMATICAL FORMALISM}

In the grain-count regime the surviving fraction of a cellular population whose radiosensitivity parameters are $m, E_{0}, \sigma_{0}$ and $\kappa$, after track-segment irradiation with an ion dose $D$ of a fluence of $F$ particles of atomic number $Z$, effective charge value $Z^{*}$, relative speed $\beta$ and stopping power $L\left(\mathrm{LET}_{\infty}\right)$, is found from the expressions

$$
N / N_{0}=\Pi_{i} \times \Pi_{\gamma}
$$

where the ion-kill mode survival probability is

$$
\Pi_{i}=\exp (-\sigma F)
$$

where the gamma-kill mode survival probability is

$$
\Pi_{\gamma}=1-\left[1-\exp \left(\frac{-D_{\gamma}}{E_{0}}\right)\right]^{m}
$$

and the gamma-kill dose fraction is

$$
D_{\gamma}=(1-P) D
$$

where

$$
\frac{\sigma}{\sigma_{0}}=P=\left[1-\exp \left(\frac{-Z^{* 2}}{\kappa \beta^{2}}\right)\right]^{m}
$$

In the track-width regime, where $P>0.98$, we take

$$
\Pi_{\gamma}=1
$$

and find $\sigma$ from the "track width" which increases linearly with $Z^{*} / \beta$ while the inactivation cross section increases with $Z^{* 2} / \beta^{2}$ up to the limit set by the maximum radial range of $\delta$-rays. This is the "thin-down" region.

To find the cross section in the track width regime, including thin-down region, a separate calculation must be made. First we must find the "target cross section" S, for targets of radius $a_{0}$ found from $\kappa$ and $E_{0}$ according to

$$
\kappa=E_{0} a_{0}^{2} \times 5 \times 10^{6} \mathrm{erg} \mathrm{cm}
$$

and having multi-target response to gamma rays characterized by $E_{0}$ and $m$ found for the cell. This must be multiplied by the ratio of the plateau value of the cellular cross section $\sigma_{0}$ to the plateau value of the target cross section $S_{0}$ to yield the cellular action cross section in the track width regime (Katz et al. 1971). In this region we make the approximation that there is no gamma kill dose, though in the outer reaches of the track width some small fraction of the energy lost by the ion is deposited in the gamma-kill mode.

To calculate RBE at a given "kill" (transformation) or survival level we use the definition

$$
R B E=\frac{D_{x}}{D}
$$

where

$$
D_{x}=-E_{0}\left\{\ln \left[1-\left(1-N / N_{0}\right)^{1 / m}\right]\right\}
$$

is the X-ray dose after which this level obtains, and $D$ is the corresponding ion dose.

All our calculations pertain to water so the ion dose is always

$$
D=F L
$$


Where "cross sections" and RBE's are calculated from the final slope of the survival curves, we refer to the cross section and the RBE as "extrapolated" and in the grain count regime we write

and

$$
\sigma_{\mathrm{ext}}=\sigma_{0} P+(1-P) L / E_{0}
$$

$$
R B E_{\text {ext }}=\left(\sigma_{0} E_{0} / L\right) P+(1-P)
$$

To calculate the effective charge value of an ion of atomic number $Z$ moving with a relative velocity $\beta$ we use the expression (Barkas, 1963)

$$
Z^{*}=Z\left[1-\exp \left(-125 \beta Z^{-2 / 3}\right)\right]
$$

We calculate the stopping power and range in water of an ion of atomic number $Z$ with the expressions

$$
L(Z, E)=L(p, E)\left[Z^{*} / Z_{p}^{*}\right]^{2}
$$

where $Z^{*}$ and $Z_{p}^{*}$ are the effective charges of the ion and proton, respectively and $L(p, E)$ is the stopping power, in water, of a proton at the same energy/nucleon, $\mathrm{E}$.

At low fluence, where ions are sufficiently far apart that inter-track effects are unlikely, we can neglect the contribution from gamma-kill. Under this circumstance the RBE is

$$
R B E=E_{0}\left(\frac{\sigma}{L}\right)^{1 / m} D^{(1 / m-1)}
$$

This is applicable to low doses of neutrons as well as the effects of galactic cosmic rays (Katz and Cucinotta, 1991).

For mixed radiation fields, our model requires knowledge of the particle-energy spectrum of the radiation field. We then find the totality of effects due to ion kill, add together the gamma kill doses including the dose from gamma rays, find the ion kill survival and the gamma kill survival probabilities, and take their product to be the surviving fraction of irradiated cells. We have done this for neutrons admixed with gamma rays, for range modulated heavy ion beams, and most recently for cosmic rays.

\section{CELL KILLING, CHROMOSOME ABERRATIONS, TRANSFORMATIONS}

Our treatment of transformations (Waligorski, Sinclair and Katz, 1987) is based on data obtained with the BEVALAC accelerator by Yang et al. (1985). We use the same form of equations as for cell killing, and take cell killing and transformation to be independent processes that take place along the same particle track. Our parameters for Chinese hamster cells are based on the data of Skarsgard et al. (1967) while the parameters for tradescantia are based on the data of Underbrink et al. (1978). Here we have attempted to extract parameters from data obtained with $\mathrm{x}$ rays and neutron irradiations of two different energy spectra, ignoring possible gamma ray contamination. The parameters for T-1 cells of human origin are taken from Todd (1967). The separate sets of parameters for survival, chromosome aberration and for transformation, are shown in Table 1 (Katz and Huang, 1991). Where two sets of parameters are shown, the data do not permit a clear distinction between them.

\section{TARGET FRAGMENTATION EFFECTS}

High energy protons passing through tissue will occasionally suffer nuclear reactions that produce low energy, high LET ions from the tissue itself. The target fragments, in turn, will be a source of delta-ray production which should contribute to biological damage locally in the tissue matrix. The differential fluence (Wilson, 1977) describes the local source of target fragments

$$
F_{j}=\frac{1}{L\left(Z_{j}, E\right)} \int_{E}^{\infty} \frac{d \Sigma_{j}\left(E^{\prime}\right)}{d E^{\prime}} F_{p}\left(E_{p}\right) d E^{\prime}
$$


where $j$ is the fragment label, $L$ the stopping power or LET, $\Sigma_{j}$ the macroscopic nuclear production cross section, and $F_{p}$ the fluence of protons with energy $E_{p}$. An effective action cross section for the proton dressed by the target fragments (nuclear stars) is now written as

$$
\sigma^{*}=\sigma_{p}\left(E_{p}\right)+\frac{1}{F_{p}\left(E_{p}\right)} \sum_{j} \int_{0}^{\infty} F_{j}\left(E_{j}\right) \sigma_{j}\left(E_{j}\right) d E_{j}
$$

where $\sigma_{p}$ and $\sigma_{j}$ are given by the Katz formalism [Eq. (5)]. The gamma-kill dose for the proton plus target fragments is written

$$
D_{\gamma}=D_{\gamma_{p}}\left(E_{p}\right)+\sum_{j} \int_{0}^{\infty} F_{j}\left(E_{j}\right) L\left(Z_{j}, E_{j}\right)\left[1-P_{j}\left(E_{j}\right)\right] d E_{j}
$$

The production energy spectra for the target fragments is expressed as (Wilson, et al., 1989)

$$
\frac{d \Sigma_{j}}{d E}=\frac{\Sigma_{j}\left(E_{p}\right) \sqrt{E}}{\left(2 \pi E_{0 j}^{3}\right)^{1 / 2}} e^{-E / 2 E_{0 j}}
$$

where $3 E_{0 j}$ is the average energy of the fragment.

The fragmentation parameters used are discussed in Wilson et al. (1989). The light ion production cross sections are from the Bertini Monte Carlo results (Bertini, 1970) and the Silberberg-Tsao empirical model is used for the heavier fragments (Silberberg, et al., 1976). The average energy of the tissue fragments is related to the momentum width measured experimentally (Greiner et al., 1975), which Wilson et al. (1989) fits empirically. We note that the largest uncertainties exist for light ion production $(A=7$ and 9$)$ and for energies below $100 \mathrm{MeV}$. Elastic recoils and meson production, above several hundred $\mathrm{MeV}$, will also

\begin{tabular}{|c|c|c|c|c|}
\hline & $m$ & $\kappa$ & $\begin{array}{l}E_{o} \\
\text { Gy }\end{array}$ & $\begin{array}{c}\sigma_{0} \\
\mathrm{~cm}^{2}\end{array}$ \\
\hline \multicolumn{5}{|l|}{$\begin{array}{l}\mathrm{CH}_{2} \mathrm{~B}_{2} \text { Chinese Hamster Cells } \\
\text { Skarsgard et al. } 1967\end{array}$} \\
\hline survival & 3 & 1100 & 1.82 & $4.3 \times 10^{-7}$ \\
\hline abnormal metaphases & 3 & 900 & 1.82 & $3 \times 10^{-7}$ \\
\hline chromatid exchanges & 2 & 1400 & 25 & $6.5 \times 10^{-9}$ \\
\hline \multicolumn{5}{|l|}{ C3H10T1/2 Mouse Cells } \\
\hline Yang et al. 1985 & & & & $5 \times 10-7$ \\
\hline survival & 3 & 750 & 1.7 & $12 \times 10^{-10}$ \\
\hline transformations & 2 & $\begin{array}{l}750 \\
475\end{array}$ & $\begin{array}{r}180 \\
50\end{array}$ & $7 \times 10^{-11}$ \\
\hline \multicolumn{5}{|l|}{ Tradescantia } \\
\hline survival & 2 & 1000 & 2.1 & $3.5 \times 10^{-7}$ \\
\hline survival & or 1.5 & 1900 & 2.6 & $4.0 \times 10^{-7}$ \\
\hline \multicolumn{5}{|l|}{ Human T-1 Cells } \\
\hline survival (aerobic) & 2.5 & 1000 & 1.7 & $6.7 \times 10^{-7}$ \\
\hline survival (hypoxic) & 2.5 & $1300(1450)$ & $4.6(5.2)$ & $6.7 \times 10^{-7}$ \\
\hline
\end{tabular}

TABLE 1. PARAMETERS FOR SURVIVAL, ABERRATIONS, TRANSFORMATIONS 
contribute and should be added. The stopping power in tissue is from the work of Wilson (1983) based on the Ziegler analysis (Ziegler, 1980).

The solid line in Fig. 1 displays the fragment LET component from 1-GeV protons in tissue derived from eq. (16). The dotted and dashed lines show the contributions from proton fragment and alpha secondaries, respectively.

The proton action cross section for cell survival of Chinese hamster cells is shown in Fig. 2 versus the proton energy. The cellular response parameters are given in Table 1. Discussed below are comparisons to the data of Hall et al. (1978) for survival of Chinese hamster cells where the characteristic $\mathrm{X}$-ray dose, $E_{0}$, is taken as $2.9 \mathrm{~Gy}$, as found from their X-ray data and with the remaining parameters the same as given by Table 1. The dotted line in Fig. 2 shows the contributions from primary ionizations; the dashed line shows contributions from secondary ions. We note that the oxygen and nitrogen fragments contribute partially to the cross section in the track-width regime. The decreasing proton LET with increasing energy is seen to lead to complete domination by target fragments above about $50 \mathrm{MeV}$. The shape of the action cross section in Fig. 2 directly reflects the nuclear absorption cross section in tissue. We expect a further increase above several hundred $\mathrm{MeV}$ when meson production is included in the cross section. In Table 2, the individual contributions to the action cross section are shown for several proton energies. Secondary protons and alphas are dominant with a broad spectra of tissue fragments making non-negligible contributions. The primary proton makes up an insignificant fraction of the action cross section above $100 \mathrm{MeV}$, and the relatively slow change with energy of the nuclear production cross sections leads to a plateau in the action cross section at high proton energies.

Figure 3 shows the action cross section versus proton LET with the calculations of Fig. 2, extended down to $0.1 \mathrm{MeV}$, corresponding to high LET protons. The behavior of the cross section below $0.5 \mathrm{keV} / \mu$ shows the dominance of the tissue secondaries (nuclear stars). At about $0.2 \mathrm{keV} / \mu$, the proton LET minimizes and then increases, which is the origin of the 'hook' in Fig. 3 at the lowest LET values. It would be interesting to test our results for the proton cross section by experiment. Results herein assume an equilibrium in the local secondary fluence spectra, and are sensitive to interface effects (Cucinotta et al., 1990) and the composition of the host media of the cell culture.

In Fig. 4 we show the proton gamma-kill dose divided by $E_{o}$ versus proton energy. The primary ionization is the dotted line and the solid line includes the effects of fragments. Secondary ion production is seen to have a negligible effect on intertrack effects, except at the highest energies where a small contribution is seen.

Cellular parameters obtained for survival and neoplastic transformations of C3H10T1/2 cells obtained from the experiments of Yang et al. (1985) are given in Table 1. We note that the large uncertainties in the transformation data of Yang should lead to a similar uncertainty in the transformation parameters. Parameter sets were found from data for instanteaneous and delayed plating of the cells after the irradiation. Here only the delayed plating case is considered. General agreement with the measured RBE values was found using these parameter sets (Waligorski et al., 1987). The single-particle-inactivation cross section neglecting the target fragmentation of eq. (17) is shown in Figs. 5 and 6 for cell death and cell transformation, respectively, as a function of the energy of the passing ion. The targetfragmentation contribution [the second term in eq. (17)] for protons has been evaluated as shown in Figs. 7 and 8. For protons the effects of the target fragments [dashed line, second term in eq. (17)] dominate over the proton direct ionization (dotted line) at high energy. For high LET particles (low energy), the direct ionization dominates and target-fragmentation effects become negligible. A simple scaling by $A_{j}^{1 / 2}$ relates the proton target-fragment term to ions of mass $A_{j}$. The resulting effective-action cross sections for cell death and cell transformation are plotted in Figs. 9 and 10, respectively. We note that the low-energy ${ }^{56} \mathrm{Fe}$ component of the GCR spectra extends into the trackwidth regime where $\sigma>\sigma_{0}$ and is not represented in the present calculations. 
TABLE 2. TARGET FRAGMENT CONTRIBUTIONS TO PROTON ACTION CROSS-SECTION FOR V79 CHINESE HAMSTER CELLS $\sigma, 10^{-12} \mathrm{~cm}^{2}$

\begin{tabular}{|c|c|c|c|c|}
\hline \multirow[b]{2}{*}{$Z f$} & \multirow[b]{2}{*}{$A f$} & \multicolumn{3}{|c|}{$E_{p}(\mathrm{MeV})$} \\
\hline & & 10 & 100 & 1000 \\
\hline 1 & 1 & 4.58 & 6.70 & 7.14 \\
\hline 1 & 2 & 0.21 & 0.40 & 1.49 \\
\hline 1 & 3 & 0.10 & 0.19 & 0.22 \\
\hline 2 & 3 & 0.16 & 0.34 & 0.66 \\
\hline 2 & 4 & 1.68 & 3.59 & 12.22 \\
\hline 3 & 5 & 0.32 & 0.42 & 0.65 \\
\hline 3 & 6 & 0.46 & 0.53 & 0.68 \\
\hline 3 & 7 & 0.04 & 0.17 & 0.42 \\
\hline 4 & 6 & 0.01 & 0.07 & 0.20 \\
\hline 4 & 7 & 0.36 & 0.42 & 0.49 \\
\hline 4 & 8 & 0.41 & 0.51 & 0.53 \\
\hline 4 & 9 & $<0.01$ & 0.04 & 0.09 \\
\hline 5 & 8 & $<0.01$ & 0.04 & 0.11 \\
\hline 5 & 9 & 0.10 & 0.30 & 0.32 \\
\hline 5 & 10 & 0.22 & 0.35 & 0.28 \\
\hline 5 & 11 & 0.02 & 0.21 & 0.35 \\
\hline 6 & 10 & $<0.01$ & 0.03 & 0.08 \\
\hline 6 & 11 & 0.04 & 0.41 & 0.32 \\
\hline 6 & 12 & 0.50 & 1.00 & 0.63 \\
\hline 6 & 13 & 0.14 & 0.32 & 0.24 \\
\hline 6 & 14 & $<0.01$ & 0.02 & 0.03 \\
\hline 7 & 12 & $<0.01$ & 0.01 & 0.04 \\
\hline 7 & 13 & 0.03 & 0.09 & 0.07 \\
\hline 6 & 14 & $<0.01$ & 0.02 & 0.03 \\
\hline 7 & 12 & $<0.01$ & 0.01 & 0.04 \\
\hline 7 & 13 & 0.03 & 0.09 & 0.07 \\
\hline 7 & 14 & 1.11 & 0.82 & 0.37 \\
\hline 7 & 15 & 0.02 & 0.24 & 0.41 \\
\hline 8 & 14 & $<0.01$ & 0.02 & 0.05 \\
\hline 8 & 15 & 0.02 & 0.56 & 0.28 \\
\hline \multicolumn{2}{|c|}{ Primary } & 32.61 & 0.05 & $<0.01$ \\
\hline \multicolumn{2}{|c|}{ Total } & 43.16 & 17.84 & 28.35 \\
\hline
\end{tabular}




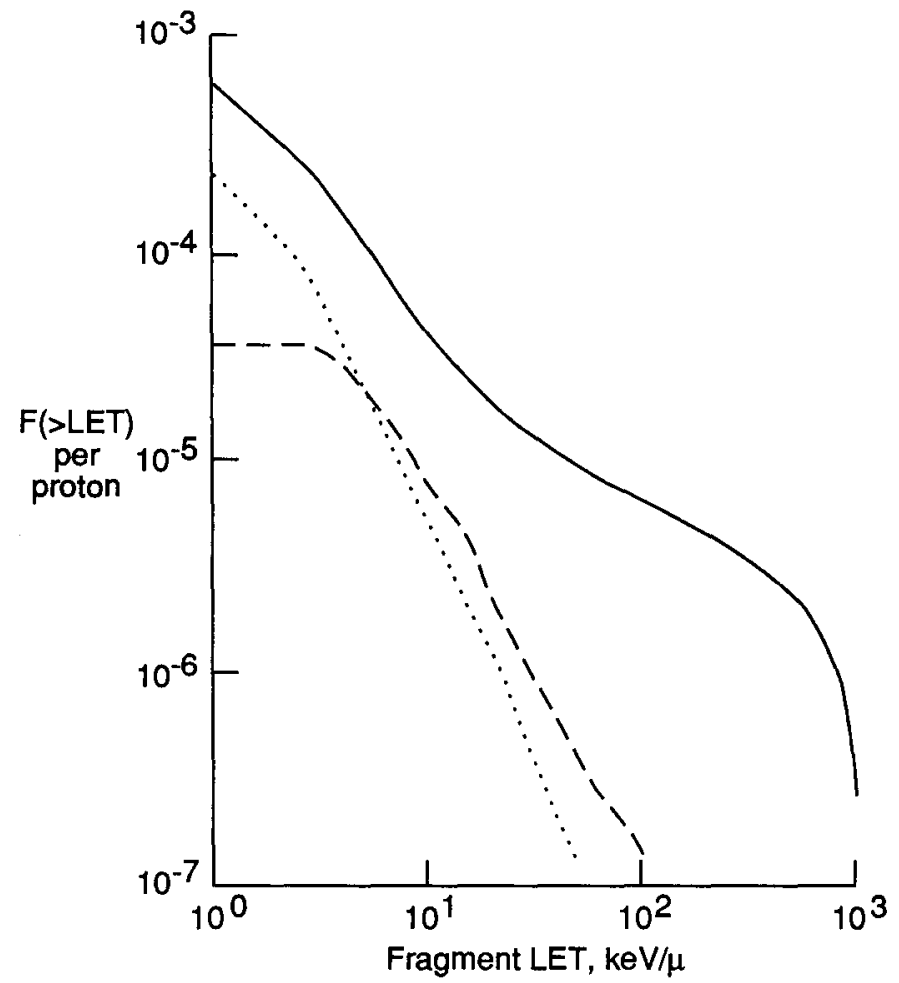

Fig. 1. Integral LET spectra for nuclear fragments produced by $1-\mathrm{GeV}$ protons in water. Solid line, all fragments; dotted line, secondary protons; and dashed line, secondary alpha particles.

\section{SURVIVAL CURVES AND THE PROTON RBE}

Cell survival curves for 10-, 100-, and $1000-\mathrm{MeV}$ protons are shown as a function of absorbed dose in Figs. 11-13. The solid line includes the target fragment terms and the dashed line neglects their contributions. Results show the importance of secondary production for increasing energy. We note, for example, that at $1000 \mathrm{MeV}$ the increase in cell death due to the fragments does not lead to substantial changes in RBE at high doses as can be calculated from Fig. 13. It is in the initial portion of the survival curves where the ion-kill mode causes large differences in RBE when compared to gamma rays. The RBE versus dose is shown in Fig. 14 with all curves including the effects of target fragmentation. We note that the proton fluence is found as $F_{p}=6.24 \mathrm{D} / \mathrm{LET}$, with $F_{p}$ in protons $/ \mu \mathrm{m}^{2}$, in Fig. 14. The rise in RBE at low dose or fluence, where single proton tracks dominate, is directly attributed to ion kill from both primary protons at $10 \mathrm{MeV}$ and nuclear fragments at the higher energies. Not shown are RBE calculations neglecting the target fragments that are nearly identical to the $10 \mathrm{MeV}$ results in Fig. 14, and are almost identical to unity for the 100 - and $1000-\mathrm{MeV}$ protons. The low dose behavior of the RBE can be seen from eq. (15), where for $m=3$ (Table 1) as found from the data of Skarsgard (1967) an RBE dependence on $D^{-2 / 3}$ is found. This effect is supported experimentally as discussed below.

\section{EFFECTIVENESS OF 160-MEV PLATEAU REGION PROTONS}

Cell survival experiments have been performed at the Harvard Cyclotron for the purpose of determining the biological effectiveness of the protons. V79 Chinese hamster cells cultured in vitro were irradiated in the plateau region of the Bragg curve and in a spread-out Bragg peak by Hall et al. (1978). Here we compared the survival measurements and RBE determinations 


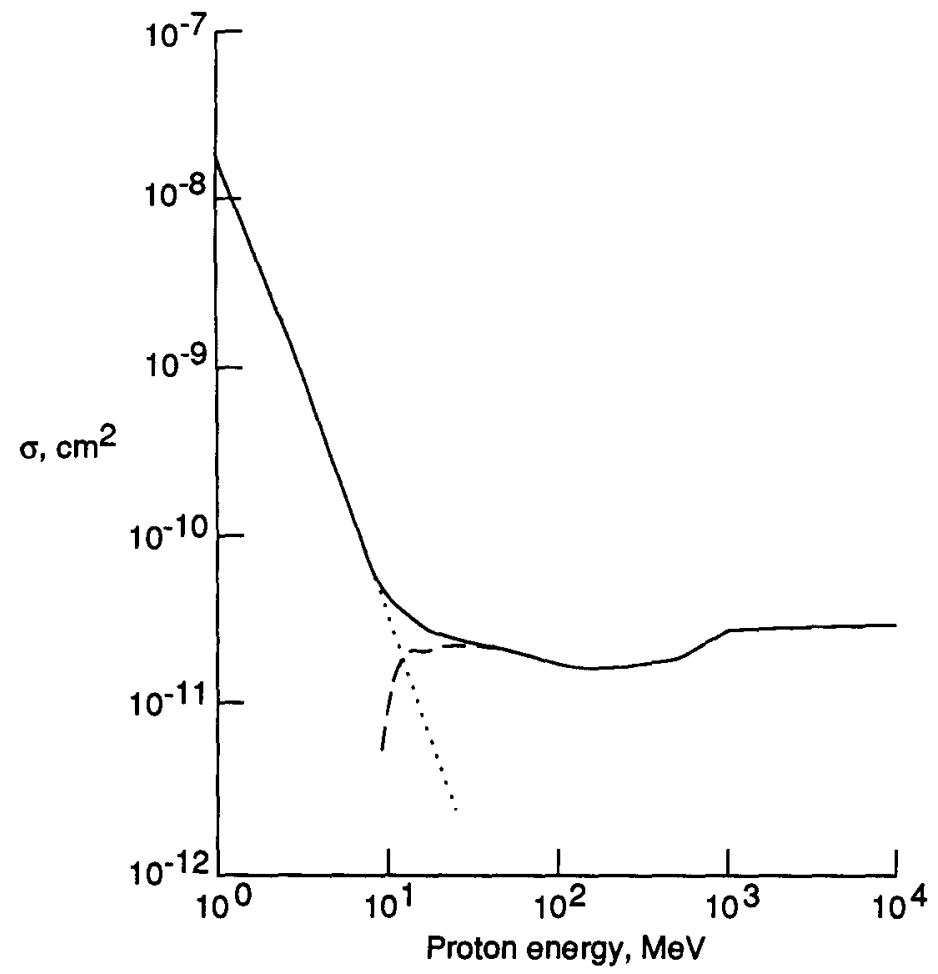

Fig. 2. Calculated values of the proton action cross section for survival of Chinese hamster cells versus the proton energy. Dotted line is primary proton contribution, dashed line is the tissue fragmentation contribution, and solid line is the total.

for attached cells in the plateau region of the $160-\mathrm{MeV}$ proton Bragg curve using the track model and the high energy nucleon transport code BRYNTRN of Wilson et al. (1989).

The nucleon transport code BRYNTRN solves the coupled proton-neutron transport problem for high energies in the straight-ahead approximation with multiple-scattering and straggling effects ignored. Target fragments with $A>1$ are transported using the production collision density as given in eq. (16). The Boltzman equations for proton and neutron transport are

$$
\left[\frac{\partial}{\partial x}-\frac{\partial}{\partial E} L\left(Z_{p}, E\right)+\Sigma_{p}(E)\right] \Phi_{p}(x, E)=\sum_{j} \int_{E}^{\infty} f_{p j}\left(E, E^{\prime}\right) \Phi_{j}\left(x, E^{\prime}\right) d E^{\prime}
$$

and

$$
\left[\frac{\partial}{\partial x}+\Sigma_{n}(E)\right] \Phi_{n}(x, E)=\sum_{j} \int_{E}^{\infty} f_{n j}\left(E, E^{\prime}\right) \Phi_{j}\left(x, E^{\prime}\right) d E^{\prime}
$$

where $\Phi_{j}$ is the differential flux of type $j$ particles at $x$ with energy $E ; L\left(Z_{j}, E\right)$ is the proton stopping power; $\Sigma_{p}(E)$ and $\Sigma_{n}(E)$ are proton and neutron total cross sections, respectively; and $f_{i j}\left(E, E^{\prime}\right)$ represents the differential cross sections for elastic and inelastic processes. As described by Wilson and Lamkin (1975) the Boltzman eqs. (20) and (21) are solved using a characteristic transformation to reduce the problem to a set of coupled integral equations with boundary conditions at $x=0$, which are then solved numerically. More details on the method of solution and the nuclear scattering data base are given by Wilson et al. (1989). The Bragg curve obtained from BRYNTRN for $160-\mathrm{MeV}$ protons in water is shown in Fig. 15 as compared to the measurements of Verhey et al. (1979) (solid line). In Fig. 15, the squares represent the primary dose and the circles the total dose with secondary production included. 


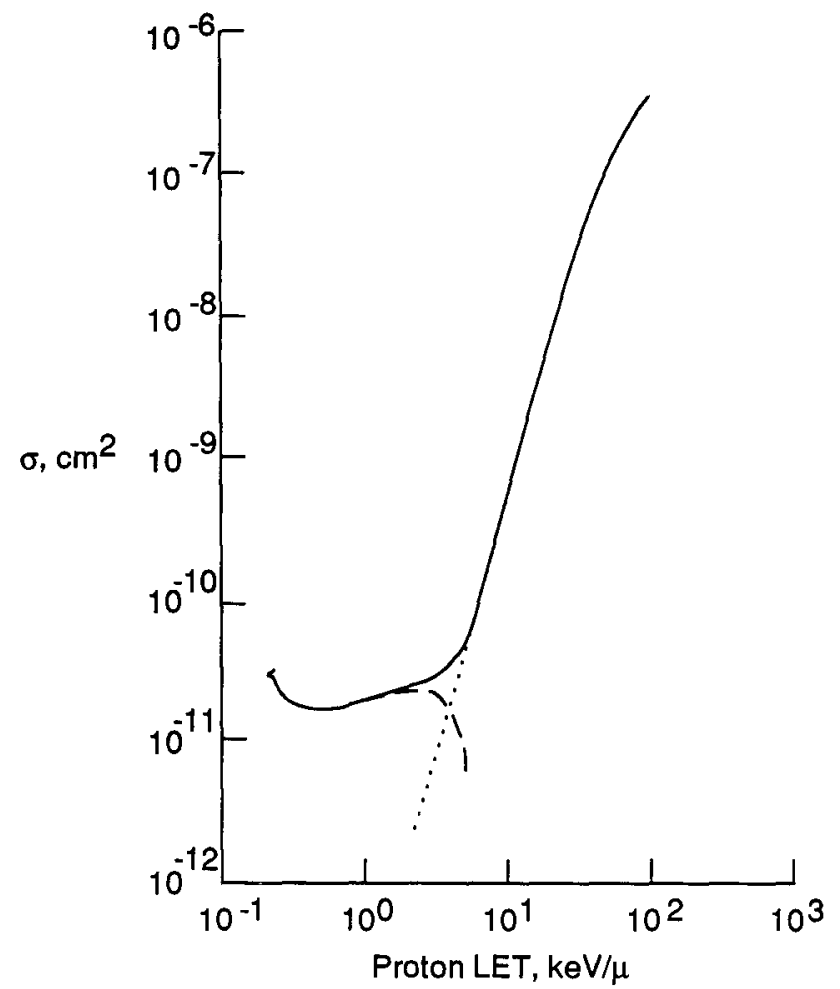

Fig. 3. Calculated values of the proton action cross section for survival of Chinese hamster cells versus the proton LET. Lines same as in Fig. 2.

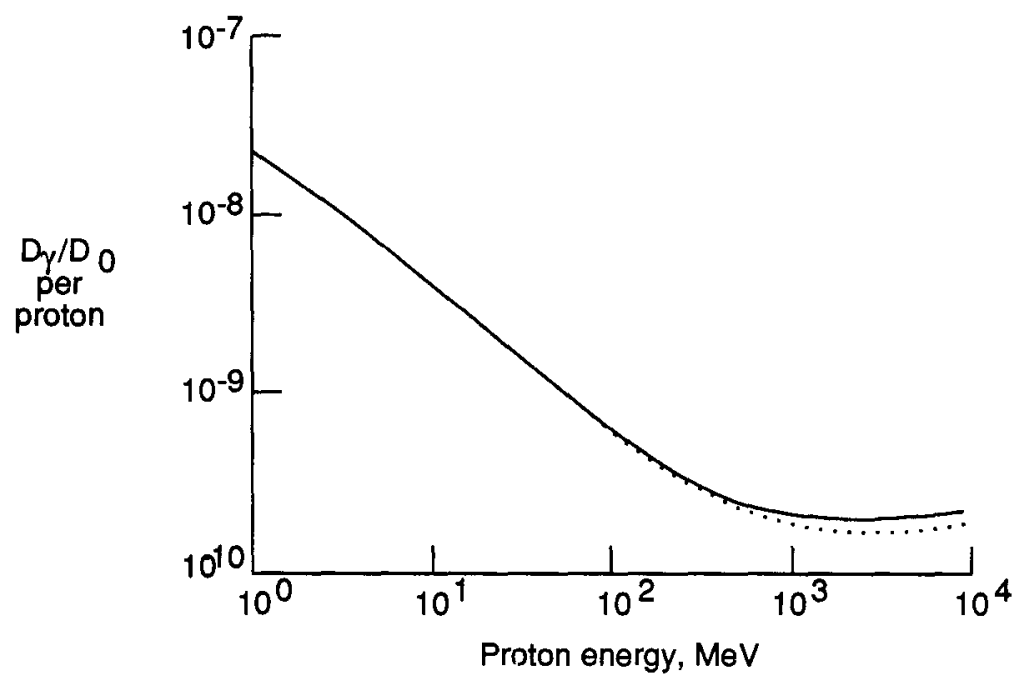

Fig. 4. Calculated values of the proton gamma-kill dose for survival of Chinese hamster cells versus the proton energy. The dotted line is the primary proton contribution and the solid includes contributions from fragmentation. 


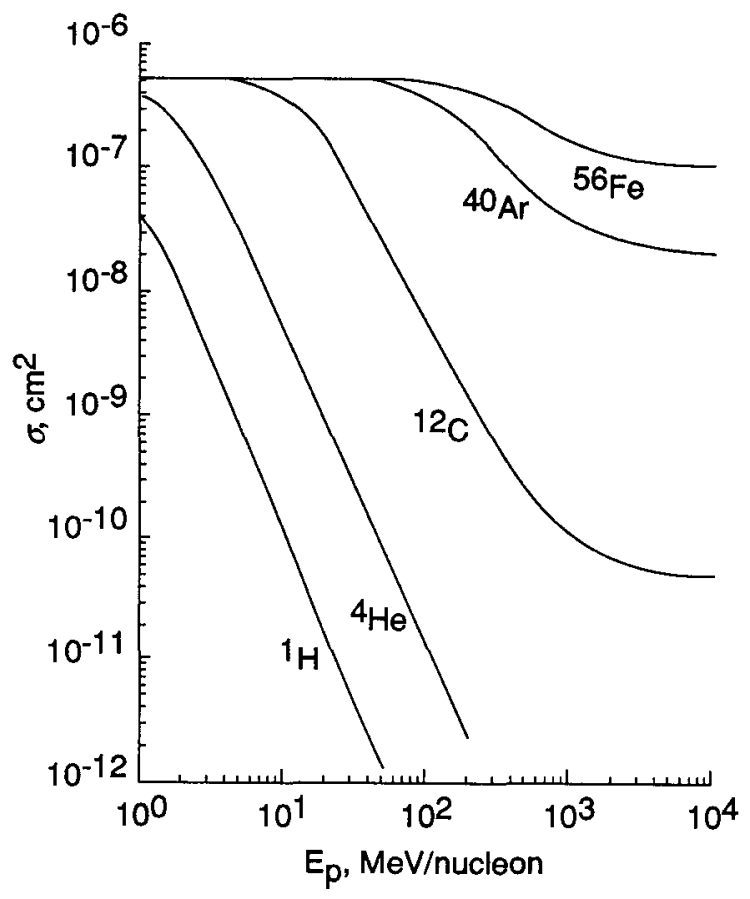

Fig. 5. Cell-death cross sections for various ions in C3H10T1/2 cells according to the Katz model for direct ionization effects only.

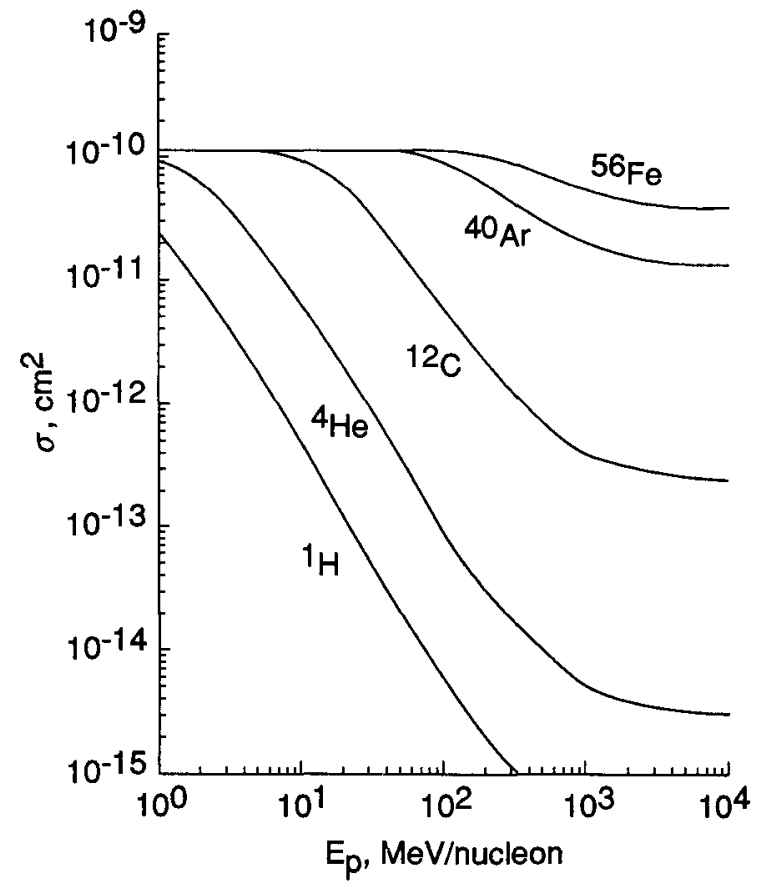

Fig. 6. Cell-transformation cross sections for various ions in $\mathrm{C} 3 \mathrm{H} 10 \mathrm{~T} 1 / 2$ cells according to the Katz model. 


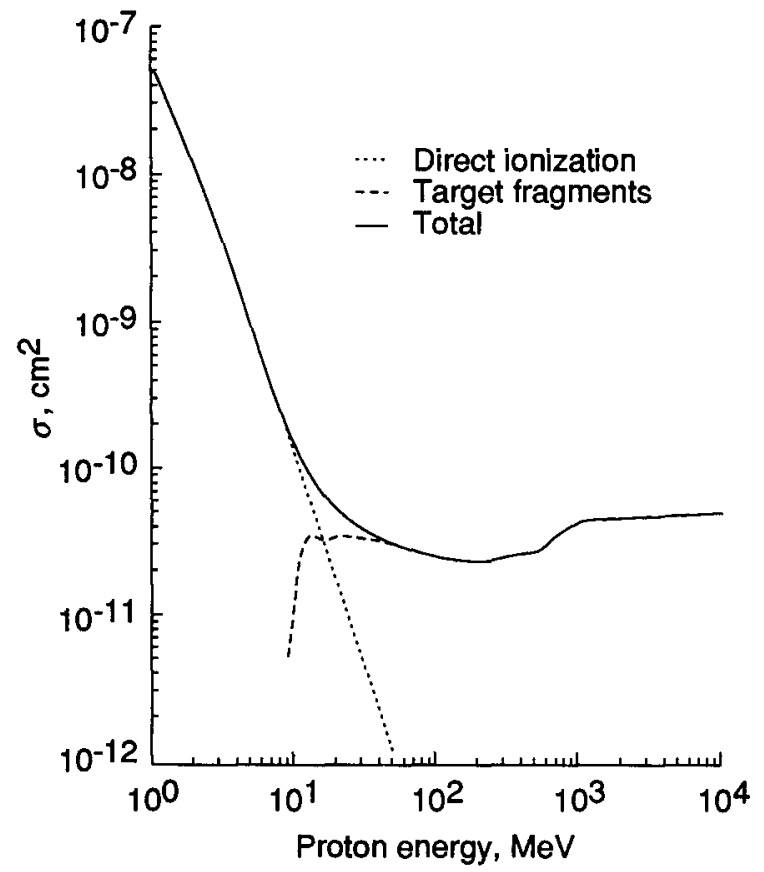

Fig. 7. Cell-death cross sections including effects of nuclear reactions for protons in C3H10T1/2 cells according to the Katz model.

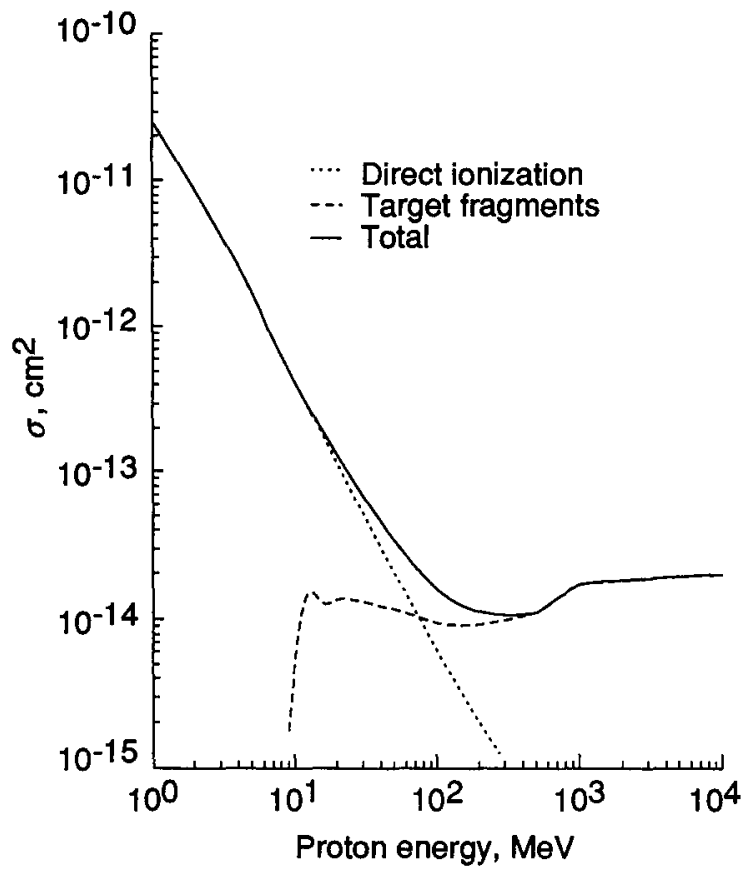

Fig. 8. Cell-transformation cross section including effects of nuclear reactions for protons in C3H10T1/2 cells according to the Katz model. 


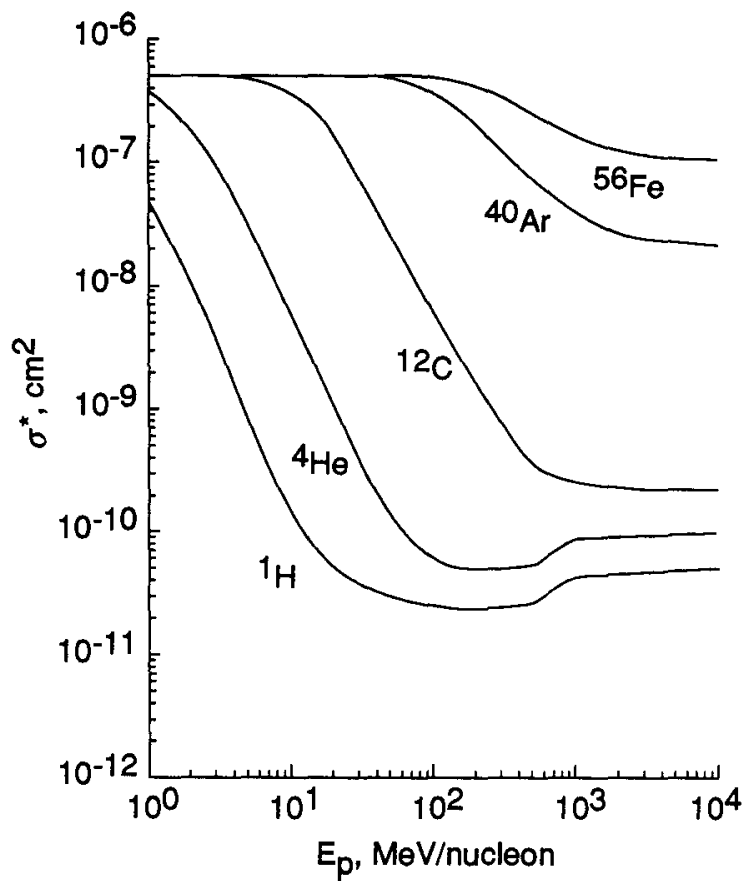

Fig. 9. Effective cell-death cross sections including effects of nuclear reactions for various ions in $\mathrm{C} 3 \mathrm{H} 10 \mathrm{~T} 1 / 2$ cells.

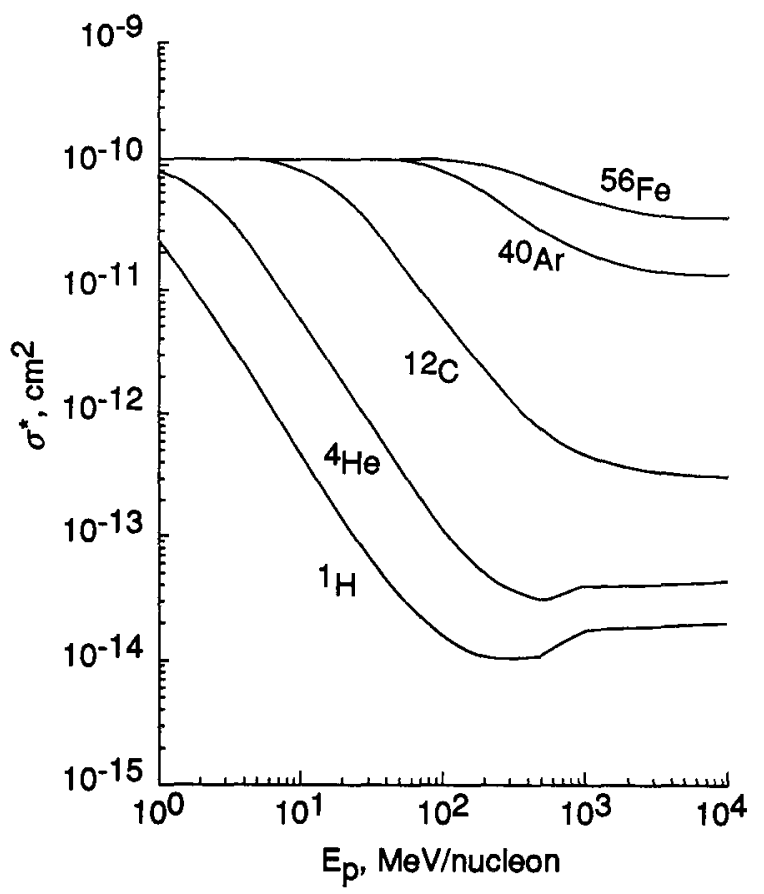

Fig. 10. Effective cell-transformation cross sections including effects of nuclear reactions for various ions in C3H10T1/2 cells. 


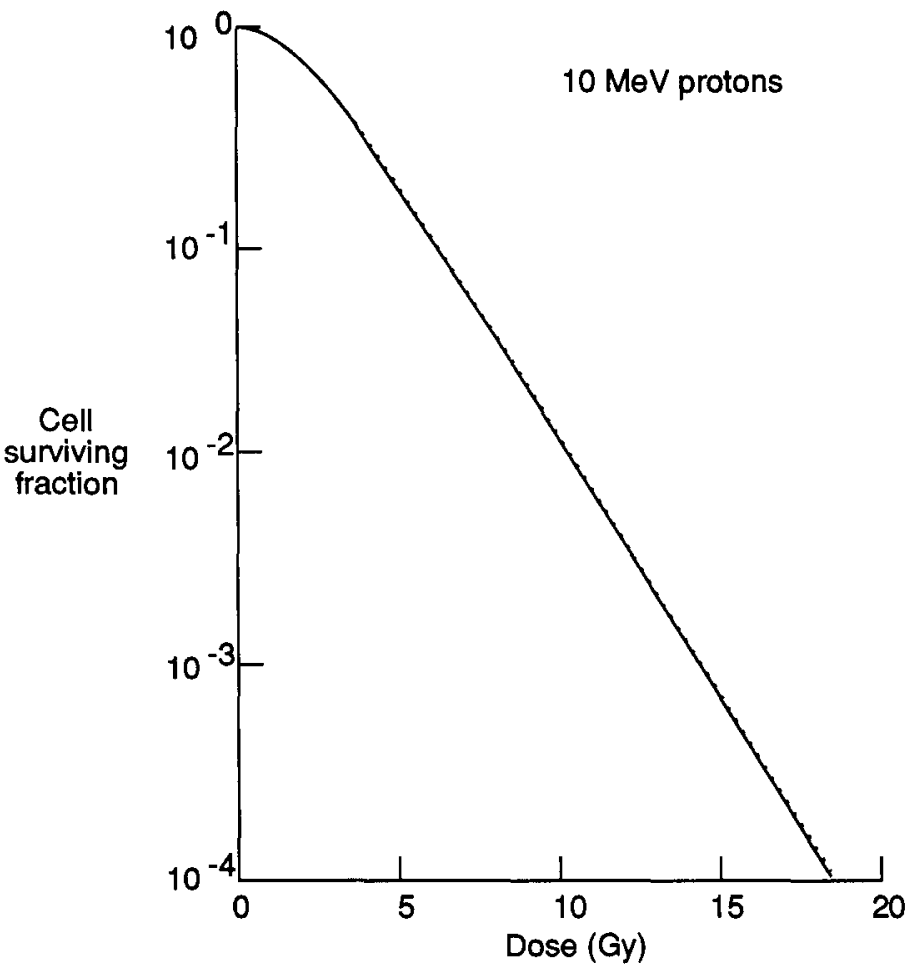

Fig. 11. Calculated cell surviving fraction of Chinese hamster cells for $10-\mathrm{MeV}$ protons. The dashed line is the primary proton and the solid adds the effects of nuclear fragments.

Calculations are normalized to the peak of the experimental Bragg curve. Straggling and multiple-scattering effects, which are not included here, both contribute significantly at the peak of the Bragg curve. We consider the plateau region where the high energy assumptions are approximately true.

At energies of $160 \mathrm{MeV}$, nuclear recoils from elastic scattering provide a sizable correction to the secondary ion production represented by target fragmentation. Elastic nuclear scattering is represented by the Born term to the optical model renormalized to the total scattering cross section in the BRYNTRN code. This representation of elastic nuclear scattering is fairly accurate for integral quantities above $100 \mathrm{MeV}$, but breaks down at lower energies because of multiple scattering, nuclear medium corrections, and especially Coulomb effects. The correction to the proton action cross section from elastic scattering is shown in Table 3 for several energies and is included in the following comparisons.

Results for the surviving fraction of suspended V79 Chinese hamster cells irradiated in the plateau region of a $160-\mathrm{MeV}$ proton beam are shown in Fig. 16. The dashed line is the fit to the $\gamma$-ray survival curve, the dotted line (barely distinguishable from the $\gamma$-response) is the contribution from primaries only, and the solid line calculations include the effects of nuclear reactions. The characteristic gamma-ray dose, $E_{o}$, is taken as $2.9 \mathrm{~Gy}$ to reproduce the experimental gamma-ray curve with the other response parameters given above. The dashed and dotted lines are nearly identical, indicating that high energy protons minus the effects of the nuclear force indeed act as gamma rays. Agreement with the data is fair indicating that the modeling of nuclear fragmentation made here is somewhat lacking. In Fig. 17 we compare our results for the proton RBE (solid line) against the values obtained using the analysis methods (vertical bars) of Kellerer and Brenot (1973). as discussed by Hall et al. (1978). The 'bare' proton RBE has a value of 1 (not shown), except at the lowest doses where a small 


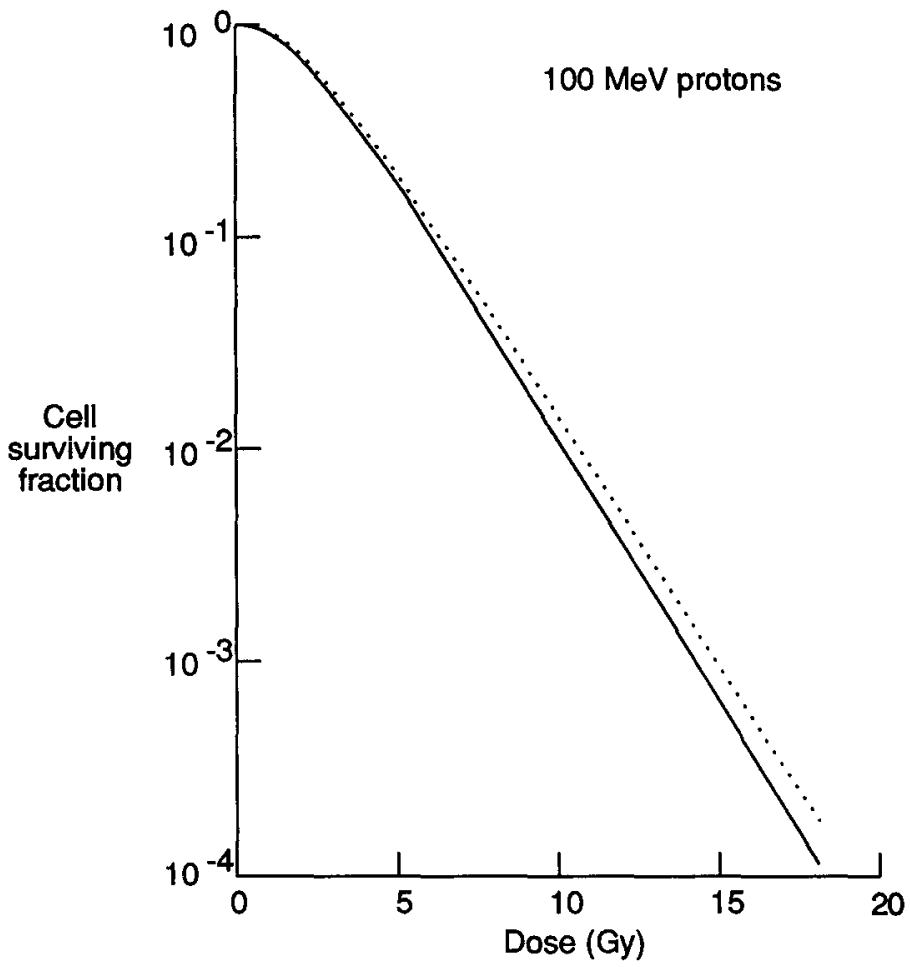

Fig. 12. Same as Fig. 11 for $100-\mathrm{MeV}$ protons.

contribution from ion kill gives a slight increase. The calculations of the proton RBE presented in Fig. 17 show good agreement with the experimentally obtained values, with the increasing $\mathrm{RBE}$ at low doses shifted to lower values than experiment. A second analysis methodology which assumes only that the dose effect curve is convex but is otherwise shape independent was used to derive RBE values (Hall et al. 1978) and are shown in Fig. 18 in comparison to the present predictions. The RBE rise at low dose as $D^{-2 / 3}$ predicted from eq. (15) is clearly seen in the calculations (solid line) and the experimental analyses (dash line). Also shown in Fig. 18 by the dotted line are our calculations neglecting nuclear reactions which are almost exactly 1 for all doses.

\section{CELL SURVIVAL IN HZE BEAMS}

The HZE transport problem has been solved and related to the Bragg curve (Wilson 1978, 1983) for monoenergetic unidirectional ion beams. The Bragg curves we calculate also provide the values for fluence estimates for the exposure conditions of biological samples yet to be analyzed. Errors in the Bragg curve translates directly into errors in exposure levels for comparisons with experimental response data.

The relative positions along the Bragg curve where biological exposures were made are indicated in Fig. 19. The survival for aerobic and hypoxic T-1 cells of human origin have been calculated using the Katz parameters in Table 1 for several locations along the beam line within a water column for three different ion beams of $\mathrm{C}, \mathrm{Ne}$ and Ar. The calculation includes both projectile as well as target fragments. The results for the $\mathrm{C}$ beam experiments (Blakeley et al. 1979) are shown in Fig. 20. The effects of overlapping $\delta$-rays are clearly apparent except near the Bragg peak where the sigmoid appearance has all but disappeared. The sigmoid returns downstream from the Bragg peak where the overlapping $\delta$-rays 


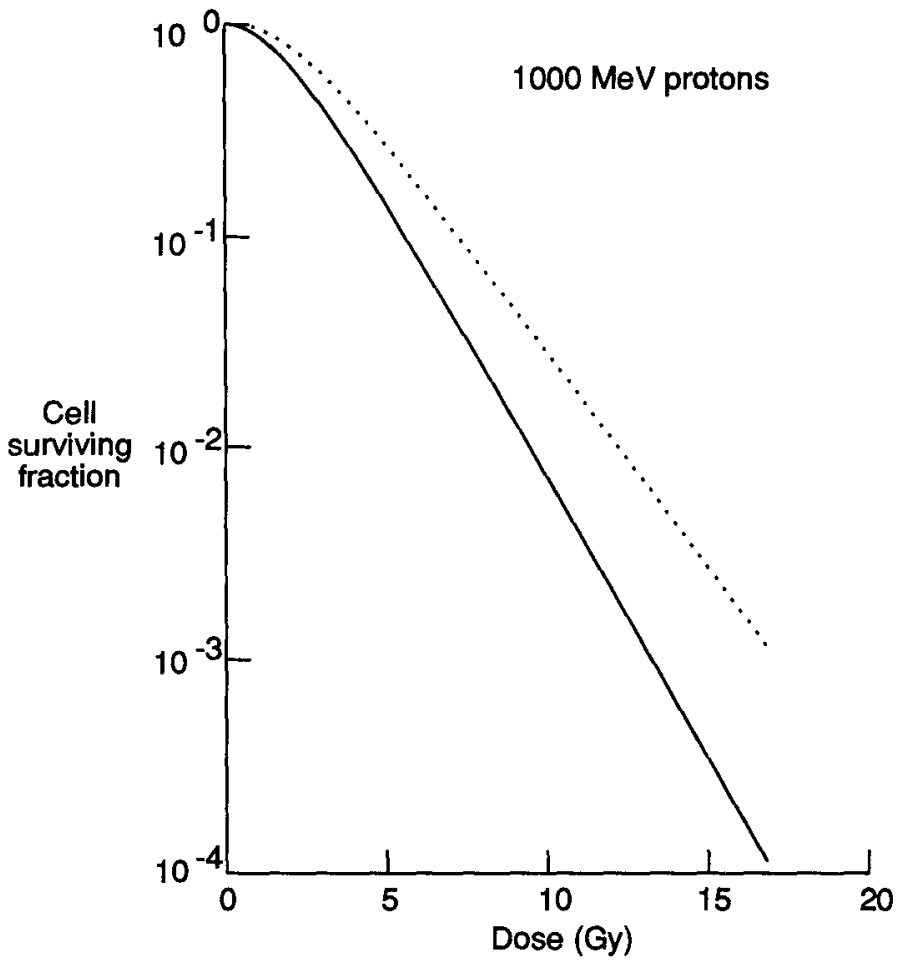

Fig. 13. Same as Fig. 12 for $1000-\mathrm{MeV}$ protons.

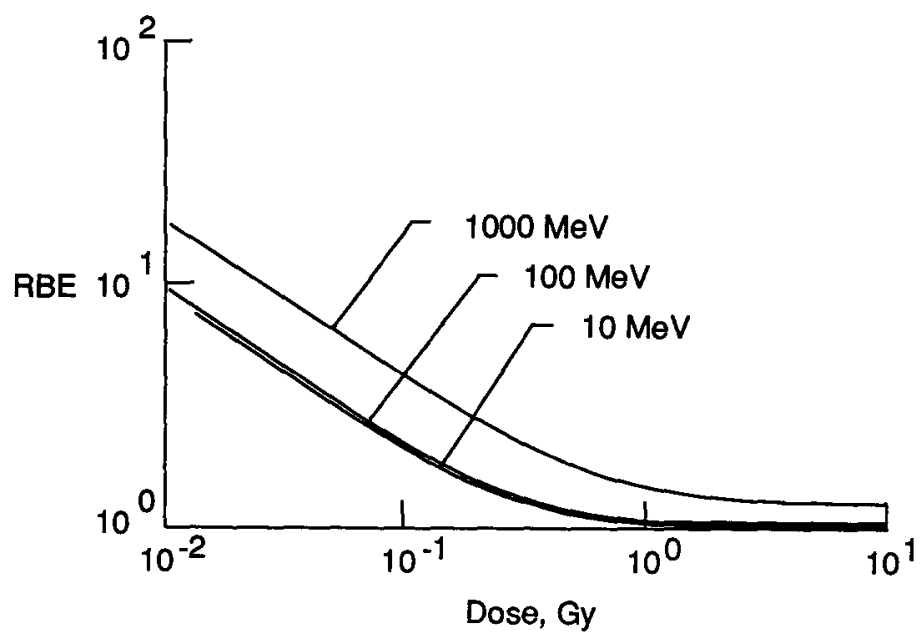

Fig. 14. Calculations of the proton RBE for survival of Chinese hamster cells versus the absorbed dose. In increasing order; 10-, 100-, 1000-MeV protons. 


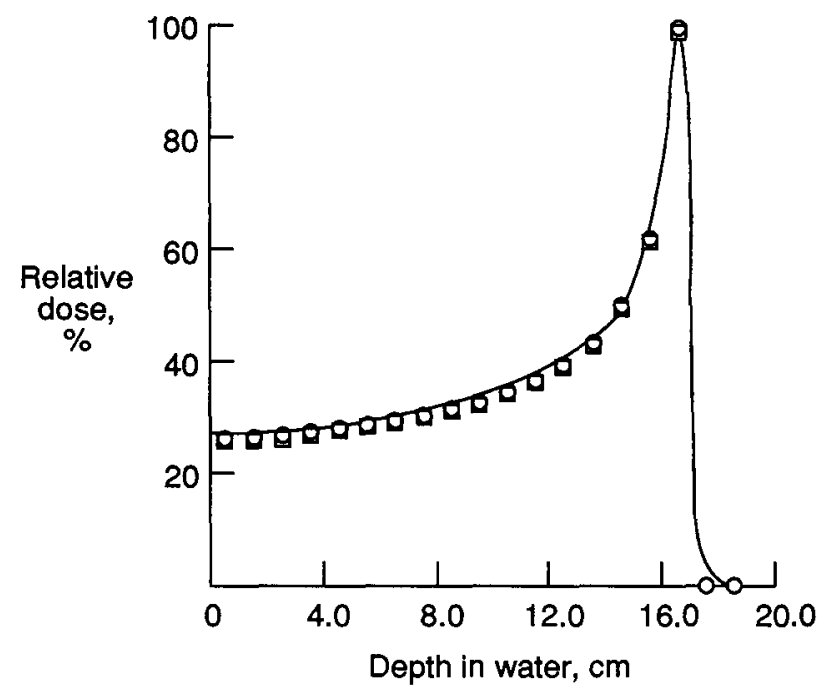

Fig. 15. Depth-dose curve for unmodulated $160-\mathrm{MeV}$ proton beam in water. The measurements of Verhey et al., 1979 (solid line) are compared to calculations with nuclear secondaries (o) and with the secondaries (a).

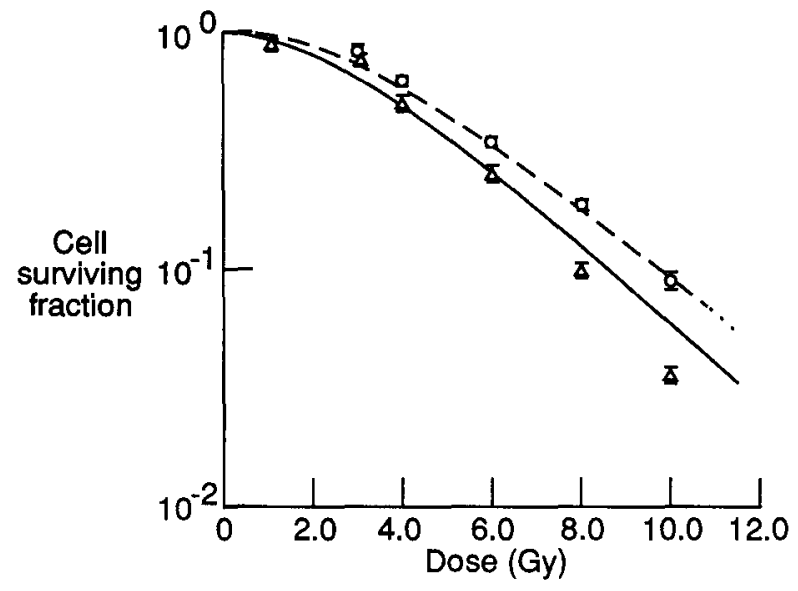

Fig. 16. Survival for Chinese hamster cells irradiated by $60-$ Co gamma rays (o) and plateau region $160-\mathrm{MeV}$ protons $(\Delta)$ from Hall et al., 1978, are compared to calculations. The dashed line is the fit to the experimental gamma-ray survival curve, the dotted line is the result for primary protons only, and the solid line includes the effects of nuclear reactions.

from adjacent ions again contribute to the exposure. Note that the oxygen effect has all but vanished near the Bragg peak while hypoxic cells show considerable radiation resistance both upstream and downstream from the Bragg peak. The results of our calculations for $\mathrm{Ne}$ beams is shown in Fig. 21. The Ne beam results are qualitatively similar to those for $\mathrm{C}$ beams. The region over which the sigmoid appearance is suppressed is greatly expanded in the Bragg peak region. The oxygen enhancement is greatly diminished one full centimeter before and after the Bragg peak as can be seen in Figs. 21. This fact is of potential importance to radiation 


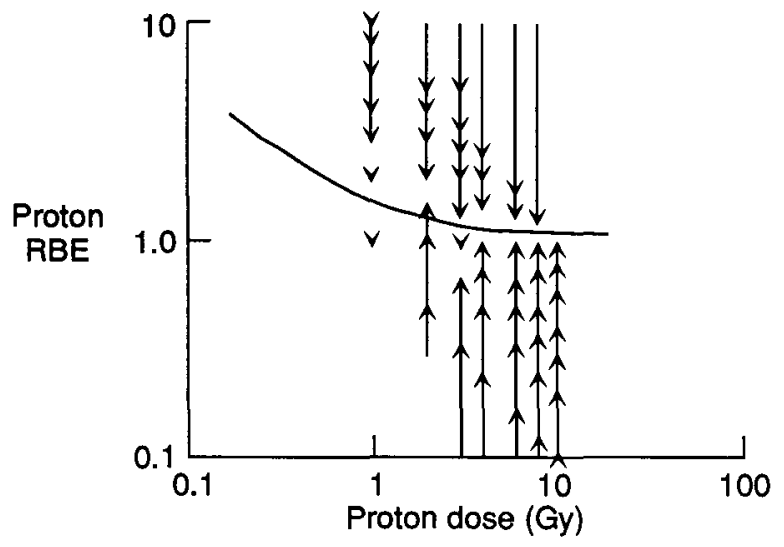

Fig. 17. Proton RBE versus proton dose for Chinese hamster cell survival in plateau region of $160-\mathrm{MeV}$ proton Bragg curve. Experimental determination from Hall et al., 1978. The vertical bars denote $\mathrm{RBE}$ values that are excluded at $95 \%$ confidence level, while the detached arrows indicate values that are unlikely at a lower level of confidence. The most likely RBE values fall in the space between the vertical bars. The solid line is from the calculations where the effects of nuclear reactions are included.

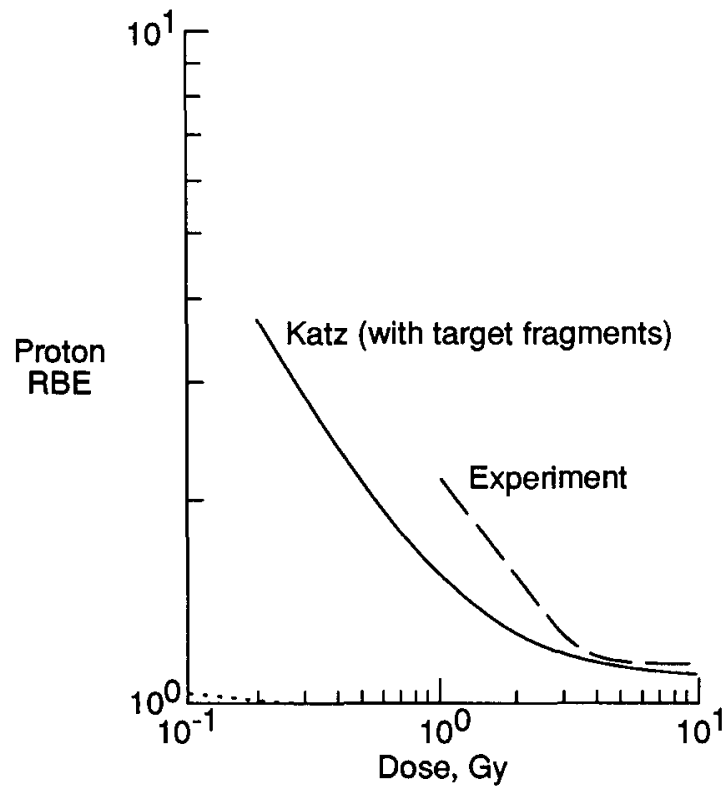

Fig. 18. Proton RBE versus proton dose for Chinese hamster cell survival in plateau region of $160-\mathrm{MeV}$ proton Bragg curve. The dashed line is from experimental analysis at ref. 10 as discussed in text. The solid line is calculations which include nuclear reactions effects, and the dotted line without nuclear reactions. 


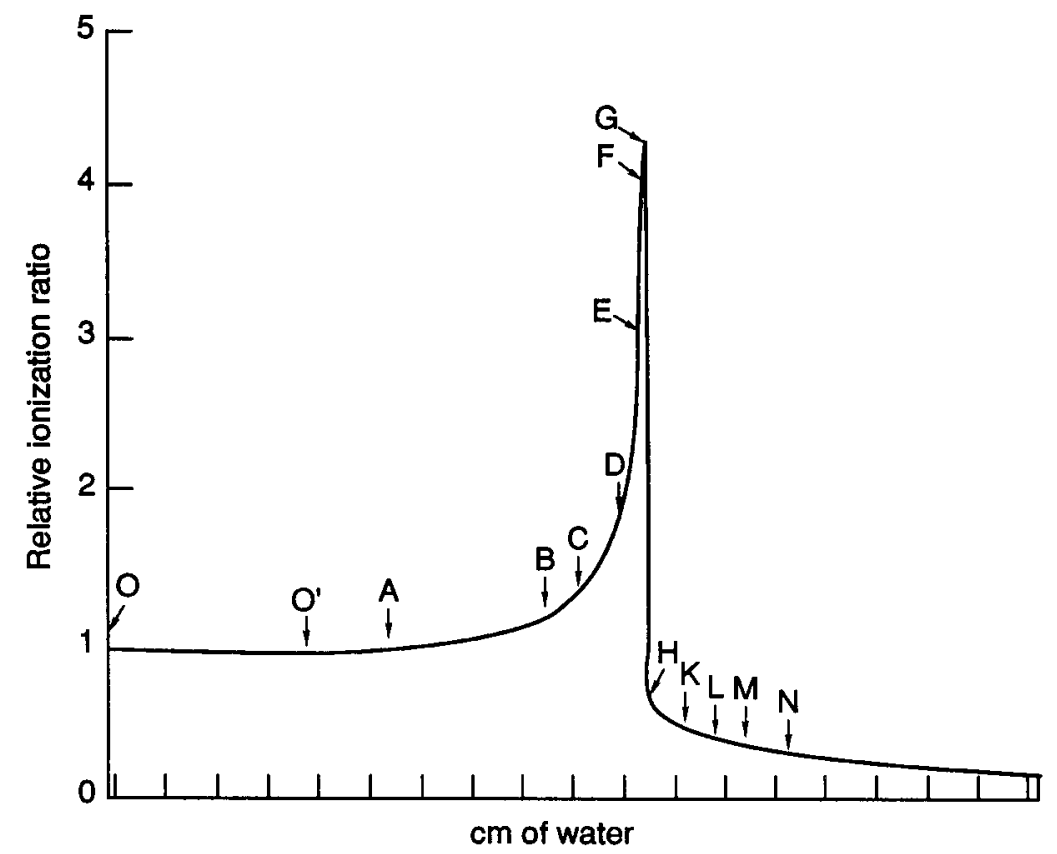

Fig. 19. Relative positions along beam line where biological experiments were performed by Blakeley et al. (1979).

therapy. The sigmoid behavior is virtually nonexistent for the $\mathrm{Ar}$ beam exposures as shown in Fig. 22. Obviously, at some great distance down stream the sigmoid shape will appear since only light fragments will survive. No experiments were conducted in this region.

\section{CELL DAMAGE FOR THE GCR SPECTRUM}

In order to apply the cellular track model to the mixed-radiation fields seen in space, we need to make the appropriate replacement of the cross section and particle fluence number $(\sigma F)$ with the particle field quantities and their corresponding cross sections. The ion-kill term, which will now contain a projectile source term (including projectile fragments) and a target fragment term, is written as

$$
\begin{aligned}
\sigma F= & \sum_{j} \int d E_{j} \Phi_{j}\left(x, E_{j}\right) \sigma_{j}\left(E_{j}\right) \\
& +\sum_{\alpha} \sum_{j} \int d E_{\alpha} d E_{j} \Phi_{\alpha}\left(x, E_{\alpha}: E_{j}\right) \sigma_{\alpha}\left(E_{\alpha}\right)
\end{aligned}
$$

where the second term is the contribution of nuclear fragments produced locally in the biological medium. This may be written in terms of an effective-action cross section $\sigma^{*}$ for the passing ion, whose track is dressed by the local target fragments (nuclear stars), as

$$
\sigma F=\sum_{j} \int d E_{j} \Phi_{j}\left(x, E_{j}\right) \sigma^{*}\left(E_{j}\right)
$$




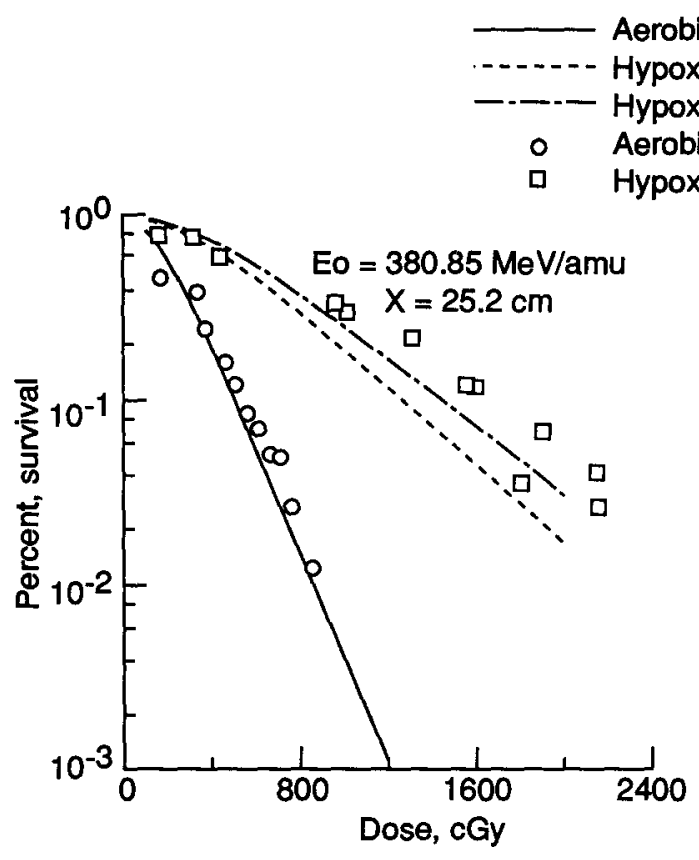

(a) $25.2 \mathrm{~cm}$ upstream.

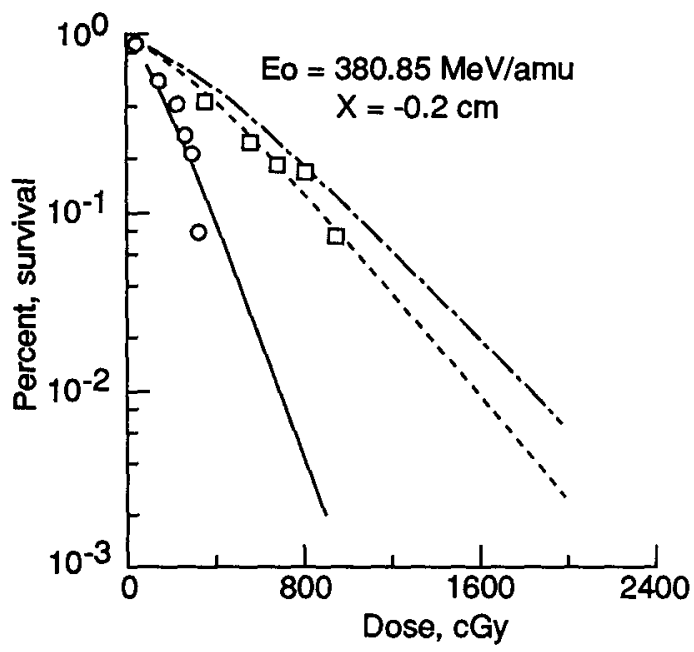

(c) $0.2 \mathrm{~cm}$ downstream.

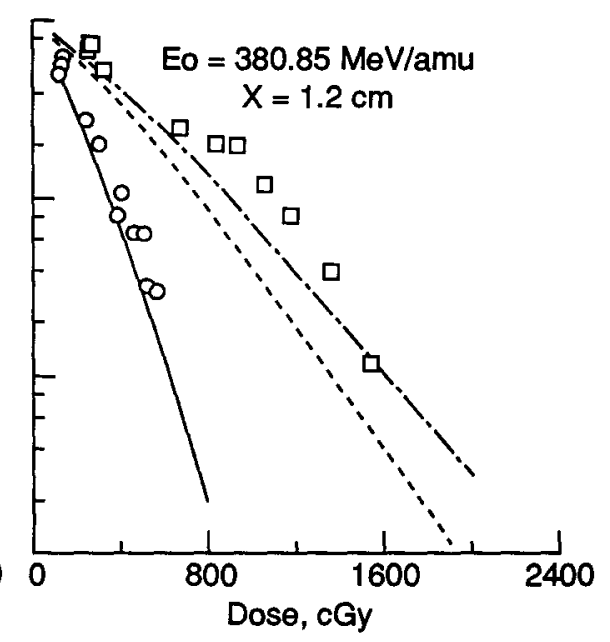

(b) $1.2 \mathrm{~cm}$ upstream.

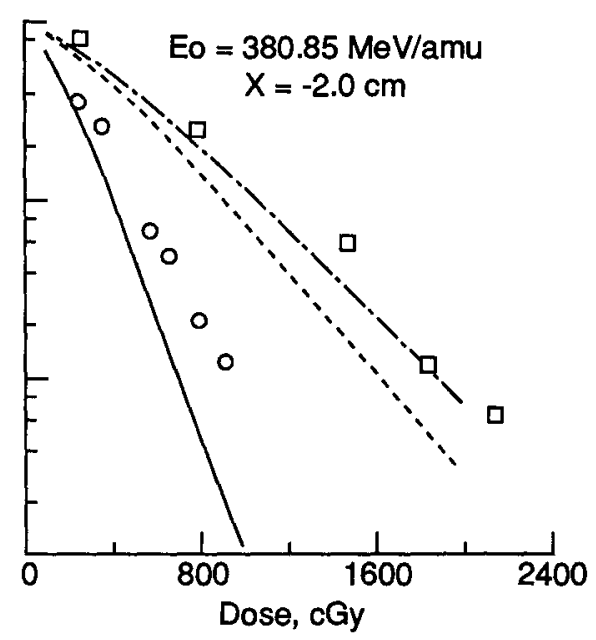

(d) $2.0 \mathrm{~cm}$ downstream.

Fig. 20. Cell survival as a function of dosage at several locations relative to the Bragg peak in $\mathrm{a}^{12} \mathrm{C}$ beam.

The gamma-kill dose fraction becomes

$$
\begin{aligned}
D_{\gamma}= & \sum_{j} \int d E_{j} \Phi_{j}\left(x, E_{j}\right)\left[1-P_{j}\left(E_{j}\right)\right] S_{j}\left(E_{j}\right) \\
& +\sum_{j} \sum_{\alpha} \int d E_{j} d E_{\alpha} \Phi_{\alpha}\left(x, E_{\alpha}: E_{j}\right) \\
& \times\left[1-P_{\alpha}\left(E_{\alpha}\right)\right] S_{\alpha}\left(E_{\alpha}\right)
\end{aligned}
$$




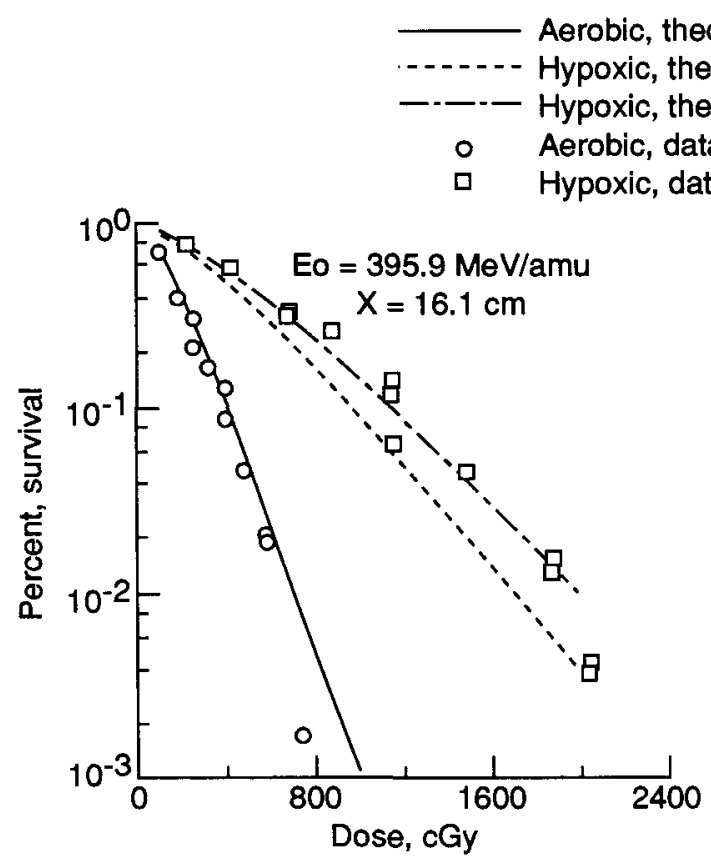

(a) $16.1 \mathrm{~cm}$ upstream.

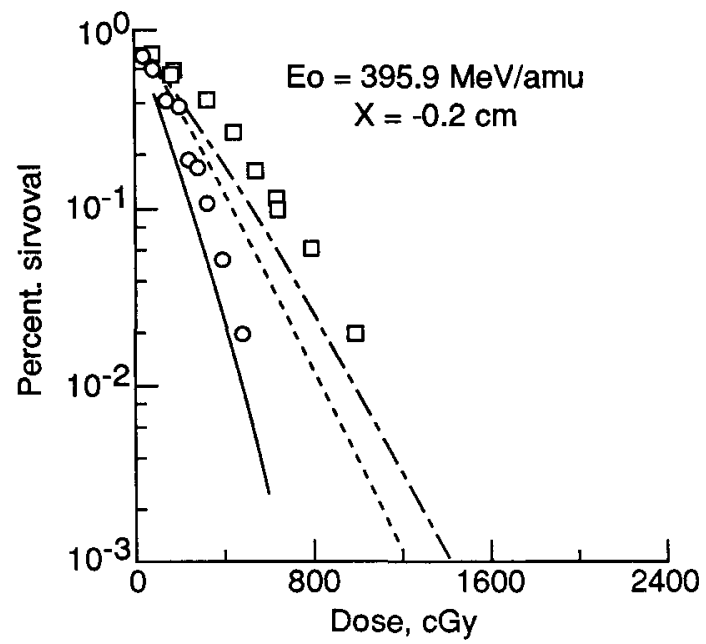

(c) $0.2 \mathrm{~cm}$ downstream.

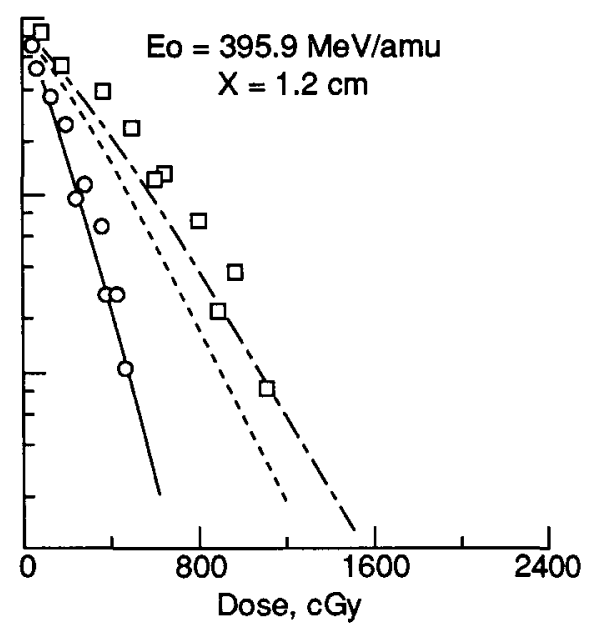

(b) $1.2 \mathrm{~cm}$ upstream.

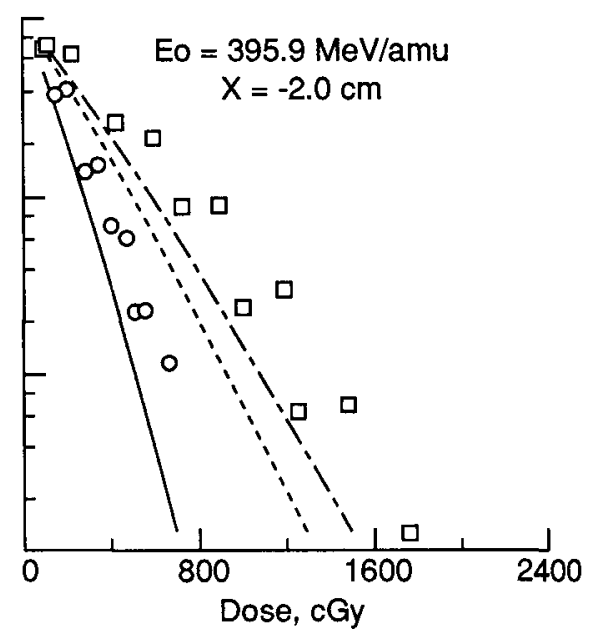

(d) $2.0 \mathrm{~cm}$ downstream.

Fig. 21. Cell survival as a function of dosage at several locations relative to the Bragg peak in a ${ }^{20} \mathrm{Ne}$ beam.

Equations (22) and (24) are used in eqs. (2) and (3), respectively. The summations over all particle types in eqs. (22) and (24) represent the addition of probabilities from all ions in the radiation field that contribute to the end point under study.

The cellular track model was applied to predict the fraction of C3H10T1/2 cells killed or transformed for 1 year in deep space at solar minimum for typical spacecraft shielding. The GCR environment was taken from the Naval Research laboratory code (Adams et al. 1981). Aluminum shielding was considered with a local region of tissue for the cell cultures. 


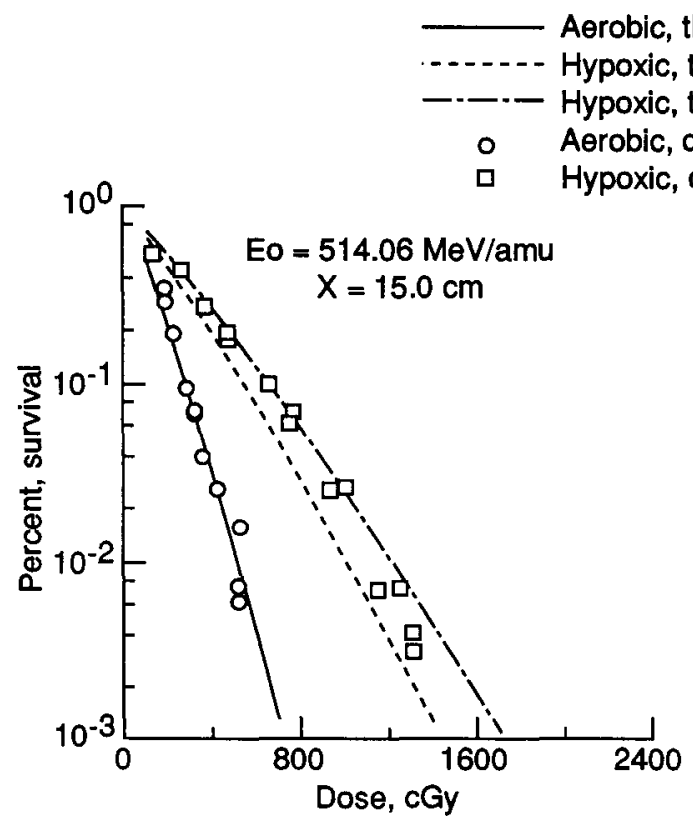

(a) $15.0 \mathrm{~cm}$ upstream.

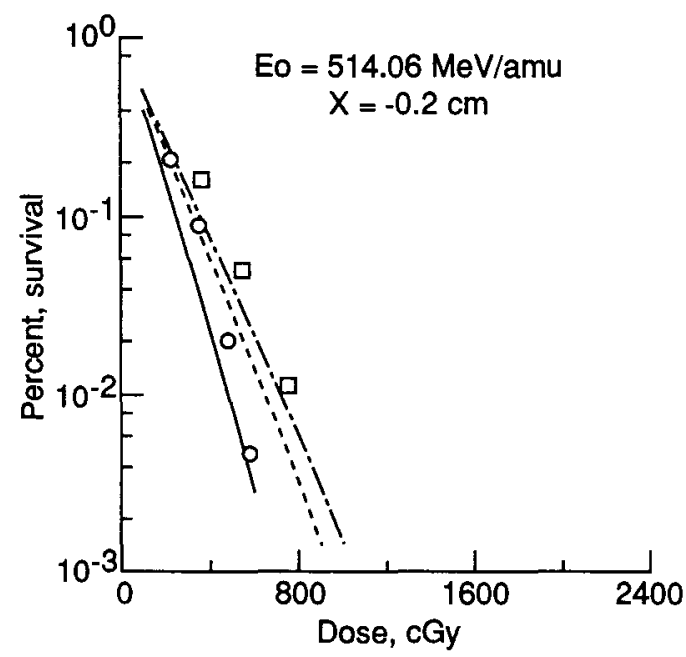

(c) $0.2 \mathrm{~cm}$ downstream.

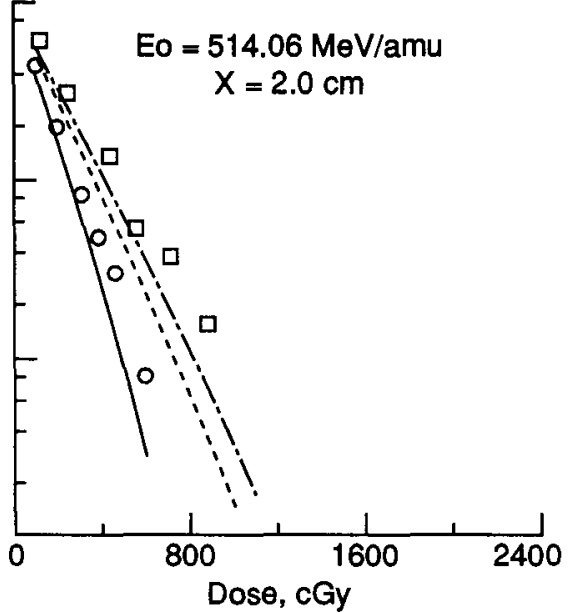

(b) $2.0 \mathrm{~cm}$ upstream.

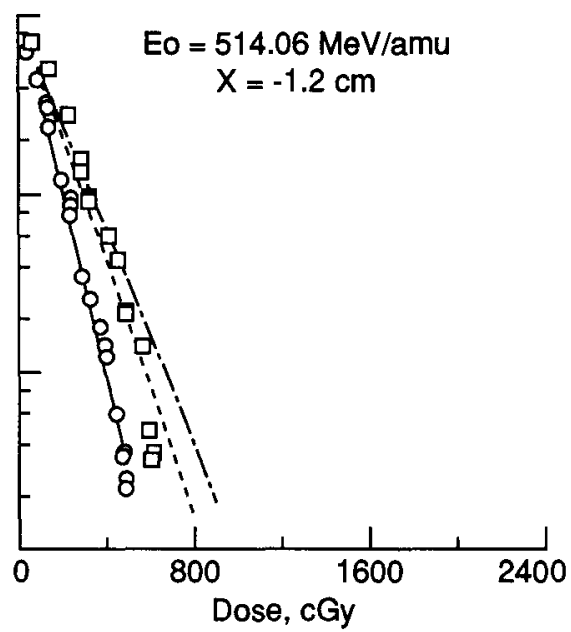

(d) $1.2 \mathrm{~cm}$ downstream.

Fig. 22. Cell survival as a function of dosage at several locations relative to the Bragg peak in a ${ }^{40} \mathrm{Ar}$ beam.

Tables 4 and 5 contain individual particle fluences and absorbed doses, respectively, for the protons, alpha particles, $Z=3$ to 9 ions (labeled H-Z) as determined by the Langley GCR code. Results for the fraction of C3H10T1/2 cells killed and transformed for 1 year at solar minimum are listed in Tables 6 and 7, respectively. The gamma-kill mode was found to be of negligible importance in the calculations, indicating that biological damage in deep space from GCR particles at the cellular level will indeed result from the action of single particles. The importance of the target terms in biological effects for low LET protons and alpha particles is quite apparent. The results also indicate that the HZE component of the GCR spectrum is 
TABLE 3. SECONDARY ION PRODUCTION CONTRIBUTION

TO PROTON ACTION ACTION CROSS SECTION FOR

V79 CHINESE HAMSTER CELLS

\begin{tabular}{|c|c|c|}
\hline \multirow{2}{*}{$E(\mathrm{MeV})$} & \multicolumn{2}{|c|}{$\sigma\left(10^{-11} \mathrm{~cm}^{2}\right)$} \\
\cline { 2 - 3 } & Elastic Recoils & Fragmentation \\
\hline 100 & 1.13 & 1.081 \\
150 & 0.82 & 1.02 \\
1000 & 0.58 & 2.09 \\
\hline
\end{tabular}

TABLE 4. FLUX FOR 1 YEAR AT SOLAR MINIMUM BEHIND ALUMINUM SHIELDING

\begin{tabular}{|c|l|l|l|l|}
\hline \multirow{2}{*}{$x, \mathrm{~g} / \mathrm{cm}^{2}$} & \multicolumn{4}{|c|}{ Flux, particles/cm $/ \mathrm{yr}$, from- } \\
\cline { 2 - 5 } & Protons & Alphas & $\begin{array}{c}\mathrm{L}-\mathrm{Z} \\
(\mathrm{a})\end{array}$ & $\begin{array}{c}\mathrm{H}-\mathrm{Z} \\
\text { (b) }\end{array}$ \\
\hline 0 & $1.29 \times 10^{8}$ & $1.24 \times 10^{7}$ & $1.09 \times 10^{7}$ & $3.0 \times 10^{7}$ \\
1 & 1.31 & 1.21 & 1.05 & 2.8 \\
2 & 1.33 & 1.18 & 1.01 & 2.7 \\
3 & 1.34 & 1.15 & 0.98 & 2.5 \\
5 & 1.36 & 1.10 & 0.91 & 2.2 \\
10 & 1.40 & 0.97 & 0.77 & 1.7 \\
20 & 1.43 & 0.77 & 0.57 & 1.1 \\
\hline
\end{tabular}

${ }^{a} Z=3$ to 9 ions.

${ }^{b} Z=10$ to 28 ions.

TABLE 5. DOSE FOR SOLAR MINIMUM BEHIND ALUMINUM SHIELDING

\begin{tabular}{|c|c|c|c|c|c|}
\hline \multirow{2}{*}{$x, \mathrm{~g} / \mathrm{cm}^{2}$} & \multicolumn{5}{|c|}{ Dose, $c \mathrm{~Gy} / \mathrm{yr}$, from } \\
\cline { 2 - 6 } & Protons & Alphas & $\begin{array}{c}\mathrm{L}-\mathrm{Z} \\
(\mathrm{a})\end{array}$ & $\begin{array}{c}\mathrm{H}-\mathrm{Z} \\
(\mathrm{b})\end{array}$ & Total \\
\hline 0 & 6.2 & 3.0 & 2.8 & 5.0 & 17.1 \\
1 & 6.3 & 2.7 & 2.5 & 3.6 & 15.1 \\
2 & 6.8 & 2.6 & 2.4 & 3.3 & 15.1 \\
3 & 7.1 & 2.6 & 2.3 & 3.1 & 15.0 \\
5 & 7.6 & 2.4 & 2.1 & 2.7 & 14.8 \\
10 & 8.5 & 2.1 & 1.7 & 2.0 & 14.3 \\
20 & 9.5 & 1.7 & 1.1 & 1.1 & 13.4 \\
\hline
\end{tabular}

${ }^{a} Z=3$ to 9 ions.

${ }^{b} Z=10$ to 28 ions. 
TABLE 6. FRACTION OF C3H10T1/2 CELLS KILLED IN DEEP SPACE FOR 1 YEAR AT SOLAR MINIMUM BEHIND ALUMINUM SHIELDING

\begin{tabular}{|c|c|c|c|c|c|c|}
\hline \multirow[b]{2}{*}{$x, \mathrm{~g} / \mathrm{cm}^{2}$} & \multicolumn{6}{|c|}{ Fraction of cells killed of - } \\
\hline & Protons & Alphas & $\begin{array}{l}\mathrm{L}-\mathrm{Z} \\
\text { (a) }\end{array}$ & $\begin{array}{r}\mathrm{H}-\mathrm{Z} \\
\text { (b) }\end{array}$ & Total & $\mathrm{RBE}$ \\
\hline \multicolumn{7}{|c|}{ Including Target Fragments } \\
\hline $\begin{array}{r}0 \\
1 \\
2 \\
3 \\
5 \\
10 \\
20\end{array}$ & $\begin{array}{l}1.35 \times 10^{-2} \\
.76 \\
.80 \\
.83 \\
.88 \\
.95 \\
1.02\end{array}$ & $\begin{array}{l}.46 \times 10^{-2} \\
.15 \\
.14 \\
.14 \\
.14 \\
.12 \\
.09\end{array}$ & $\begin{array}{l}.57 \times 10^{-2} \\
.43 \\
.41 \\
.38 \\
.34 \\
.25 \\
.15\end{array}$ & $\begin{array}{l}2.08 \times 10^{-2} \\
1.84 \\
1.69 \\
1.55 \\
1.32 \\
0.91 \\
0.49\end{array}$ & $\begin{array}{l}4.46 \times 10^{-2} \\
3.18 \\
3.04 \\
2.90 \\
2.68 \\
2.22 \\
1.74\end{array}$ & $\begin{array}{l}7.1 \\
7.0 \\
6.9 \\
6.8 \\
6.7 \\
6.5 \\
6.2\end{array}$ \\
\hline \multicolumn{7}{|c|}{ Without Target Fragments } \\
\hline $\begin{array}{r}0 \\
1 \\
2 \\
3 \\
5 \\
10 \\
20\end{array}$ & $\begin{array}{l}.84 \times 10^{-2} \\
.24 \\
.28 \\
.31 \\
.35 \\
.42 \\
.49\end{array}$ & $\begin{array}{l}.37 \times 10^{-2} \\
.06 \\
.06 \\
.06 \\
.06 \\
.05 \\
.04\end{array}$ & $\begin{array}{l}.55 \times 10^{-2} \\
.41 \\
.39 \\
.37 \\
.33 \\
.24 \\
.14\end{array}$ & $\begin{array}{l}2.08 \times 10^{-2} \\
1.83 \\
1.68 \\
1.55 \\
1.31 \\
0.91 \\
0.48\end{array}$ & $\begin{array}{l}3.79 \times 10^{-2} \\
2.54 \\
2.41 \\
2.27 \\
2.04 \\
1.61 \\
1.15\end{array}$ & $\begin{array}{l}6.7 \\
6.5 \\
6.3 \\
6.2 \\
6.1 \\
5.7 \\
5.3\end{array}$ \\
\hline
\end{tabular}

${ }^{a} Z=3$ to 9 ions.

${ }^{b} Z=10$ to 28 ions.

TABLE 7. FRACTION OF C3H1OT1/2 CELLS TRANSFORMED

IN DEEP SPACE FOR 1 YEAR AT SOLAR MINIMUM BEHIND ALUMINUM SHIELDING

\begin{tabular}{|c|l|l|l|l|l|l|l|}
\hline \multirow{2}{*}{$x, \mathrm{~g} / \mathrm{cm}^{2}$} & \multicolumn{6}{|c|}{ Fraction of cells transformed- } \\
\cline { 2 - 8 } & Protons & Alphas & $\begin{array}{c}\text { L-Z } \\
(\mathrm{a})\end{array}$ & $\begin{array}{c}\mathrm{H}-\mathrm{Z} \\
(\mathrm{b})\end{array}$ & Total & RBE \\
\hline \multicolumn{6}{|c|}{ Including Target Fragments } \\
\hline 0 & $5.2 \times 10^{-6}$ & $2.0 \times 10^{-6}$ & $3.1 \times 10^{-6}$ & $7.5 \times 10^{-6}$ & $1.78 \times 10^{-5}$ & 6.4 \\
1 & 3.5 & 1.0 & 2.7 & 6.7 & 1.39 & 6.4 \\
2 & 3.7 & 1.0 & 2.6 & 6.2 & 1.35 & 6.3 \\
3 & 3.9 & 0.9 & 2.4 & 5.7 & 1.29 & 6.3 \\
5 & 4.2 & 0.9 & 2.2 & 4.9 & 1.22 & 6.2 \\
10 & 4.7 & 0.8 & 1.7 & 3.5 & 1.06 & 6.0 \\
20 & 5.2 & 0.6 & 1.1 & 2.0 & .88 & 5.7 \\
\hline \multicolumn{6}{|c|}{ Without Target Fragments } & & \\
\hline 0 & $3.2 \times 10^{-6}$ & $1.6 \times 10^{-6}$ & $3.1 \times 10^{-6}$ & $7.5 \times 10^{-6}$ & $1.53 \times 10^{-5}$ & 6.0 \\
1 & 1.4 & 0.6 & 2.7 & 6.7 & 1.13 & 5.8 \\
2 & 1.6 & 0.6 & 2.5 & 6.2 & 1.09 & 5.7 \\
3 & 1.8 & 0.6 & 2.4 & 5.7 & 1.05 & 5.6 \\
5 & 2.1 & 0.5 & 2.1 & 4.9 & 0.97 & 5.4 \\
10 & 2.5 & 0.5 & 1.6 & 3.5 & 0.82 & 5.2 \\
20 & 3.0 & 0.4 & 1.0 & 0.64 & 4.9 \\
\hline
\end{tabular}

${ }^{a} Z=3$ to 9 ions.

${ }^{b} Z=10$ to 28 ions. 
most damaging for small shielding depths. At large depths the HZE components break up and cause proton buildup with increasing shield depth. At large depths, the protons dominate the biological effects. In comparing individual charge components, we see that the H-Z particles have a reduced effectiveness for the transformation end point.

Also listec in Tables 6 and 7 are the values of RBE versus depth for the two end points. In Table 8 we present the present $R B E$ values beside the average $Q F$ values taken from (Townsend et al. 1990) using the same transport code. The fact that RBE and QF are nearly equal at small depths is somewhat coincidental. We note that the quality factor is independent of the fluence level, which is not true for the Katz model. The Katz model indicates a substantial increase in risk, at higher shielding levels, than the ICRP 26 quality factors (ICRP 1977).

The RBE values show a simple scaling with exposure time for the GCR particles as can be seen from eqs. ( 8$),(9)$, and (2) when ion kill dominates. Here we find for

$$
\frac{N}{N_{0}} \cong 1
$$

with

$$
\sigma F \ll 1
$$

that

$$
\mathrm{RBE}=\frac{E_{o}}{\mathrm{LET}} \sigma^{1 / m} F^{[-1+(1 / m)]}
$$

Then, scaling the RBE as a function of duration in deep space to the 1-year value for a duration period of $\tau$ (with $F=n \tau$ ) gives

$$
\operatorname{RBE}(\tau)=\left(\tau / \tau_{1}\right)^{(-1+1 / m)} \operatorname{RBE}\left(\tau_{1}\right)
$$

As a result, a one-hit ( $m=1$ ) system RBE becomes fluence independent as expressed by

$$
\operatorname{RBE}(\tau)=\operatorname{RBE}\left(\tau_{1}\right)
$$

a two-hit $(m=2)$ system is expressed by

$$
\operatorname{RBE}(\tau)=\frac{\operatorname{RBE}\left(\tau_{1}\right)}{\left(\tau / \tau_{1}\right)^{1 / 2}}
$$

and a three-hit $(m=3)$ system is expressed by

$$
\operatorname{RBE}(\tau)=\frac{\operatorname{RBE}\left(\tau_{1}\right)}{\left(\tau / \tau_{1}\right)^{2 / 3}}
$$

Results of this scaling approximation agree quite well with calculations using the Katz model, as seen in Table 9 where values obtained using the approximations of eq. (27) are shown in parentheses as scaled from the 1-year RBE values taken from Table 8, and results of the calculations are shown without parentheses. The extremely large RBE values that would be obtained for small values of $\tau$ are due to the choice of energetic photons as the reference radiation.

\section{SUMMARY AND CONCLUSIONS}

Over the past 25 years Katz and coworkers developed a model of particle tracks which began with nuclear emulsions and subsequently was extended to other detectors and to the biological effects of high LET radiations. That model requires as input information knowledge of the particle-energy spectrum of the radiation environment as well as the dose of gamma rays. Calculations of the effects of beams of protons, of heavy ions, and of energetic neutrons have been hindered because of a lack of a model of such beams which included both 


\section{TABLE 8. COMPARISON OF ICRP 26 QUALITY \\ FACTORS VERSUS RBE FOR CELL DEATH AND TRANSFORMATION}

[One Year in Deep Space at Solar Minimum]

\begin{tabular}{|c|c|c|c|}
\hline$x, \mathrm{~g} / \mathrm{cm}^{2}$ & QF & $\begin{array}{c}\text { RBE for cell } \\
\text { death }\end{array}$ & $\begin{array}{c}\text { RBE for cell } \\
\text { transformation }\end{array}$ \\
\hline 0 & 7.1 & 7.1 & 6.4 \\
1 & 5.6 & 7.0 & 6.4 \\
2 & 5.3 & 6.9 & 6.3 \\
3 & 5.1 & 6.8 & 6.3 \\
5 & 4.7 & 6.7 & 6.2 \\
10 & 3.9 & 6.5 & 6.0 \\
20 & 3.2 & 6.2 & 5.7 \\
\hline
\end{tabular}

\section{TABLE 9. RBE FOR CELL DEATH AND TRANSFORMATION OF C3H10T1/2 CELLS FOR GCR SPECTRUM AT SOLAR MINIMUM BEHIND ALUMINUM SHIELDING*}

\begin{tabular}{|c|c|c|c|}
\hline \multirow{2}{*}{$x, \mathrm{~g} / \mathrm{cm}^{2}$} & \multicolumn{3}{|c|}{ RBE values for time periods of- } \\
\cline { 2 - 4 } & 1 month & 1 year & 2 years \\
\hline \multicolumn{4}{|c|}{ Cell death } \\
\hline 0 & $33.2(37.0)$ & 7.1 & $4.8(4.6)$ \\
3 & $33.2(36.1)$ & 7.0 & $4.7(4.5)$ \\
\hline \multicolumn{4}{|c|}{ Cell Transformation } \\
\hline 0 & $32.4(35.1)$ & 6.8 & $4.5(4.3)$ \\
\hline 1 & $22.3(22.2)$ & 6.4 & $4.6(4.5)$ \\
3 & $22.0(22.2)$ & 6.3 & $4.5(4.5)$ \\
\hline
\end{tabular}

*Values in parentheses scaled from 1 year value using eq. (18).

projectile and target fragmentation. A beam model created through the efforts of John Wilson $(1977 \mathrm{~b}, 1983)$ and his collaborators at NASA Langley Research Center remedied this neglect. Through it we have been able to validate both the track theory of biological effects and the beam model by comparison of our calculated radiobiological end points with ground based measurements for proton and heavy ion beams. Based on this validation we have initiated calculations of biological effects in space vehicles in selected orbits, incorporating knowledge of the distribution of solar and galactic cosmic rays to be encountered there (Cucinotta et al. $1990,1991 \mathrm{a}, 1991 \mathrm{~b}$ ). We know of no other way to estimate the biological damage in space flight at the very low fluences of heavy ions to be encountered there.

The track structure model with the deterministic galactic cosmic ray (GCR) transport code predicts the fractions of cell death and neoplastic transformations for C3H10 T1/2 cells in deep space behind typical spacecraft shielding. Results indicate that the level of damage from the GCR particles does not attenuate appreciably for large amounts of spacecraft shielding and that single particles acting in the ion-kill mode dominate the effects. The contribution from target fragments was seen to be important in assessing the biological effect of protons and alpha particles. The relative biological effectiveness (RBE) values obtained in this fluence

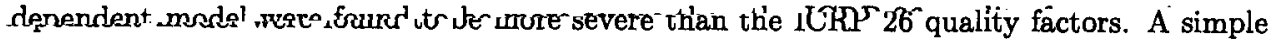


scaling law with the duration time in space was found to account for the change in RBE with fluence for the uniform GCR background.

The results of our calculations of the RBE for both cell death and cell transformation are remarkably close, especially when considering the very large difference in radiosensitivity parameters for these end points and the huge difference in the fraction of affected cells. About 1000 times as many cells are killed as are transformed. Nevertheless, 90 percent of the cells survive the conditions calculated here, and of these about 1 or 2 in 100000 are transformed. Yet, this is not an insignificant fraction when we consider the number of cells per cubic centimeter in tissue and speculate about the number of cells transformed by radiation that are likely to lead to cancer.

The cell population in tissue, about $10^{9}$ per cubic centimeter, suggests that after 1 year of exposure to GCR at solar minimum there would be about $10^{4}$ transformed cells per cubic centimeter in tissue if in vitro and in vivo transformation parameters are equal. Additionally, we do not know the minimum number of transformed cells that can be injected into a mouse to induce a cancer. Clearly, priority must be assigned to the investigation of these questions. If one or two transformed cells were to lead to cancer, as in leukemia, we could not tolerate an exposure in which the transformation fraction exceeded $10^{-9}$.

\section{ACKNOWLEDGMENT}

Research at the University of Nebraska is supported by the U.S. Department of Energy. Support for one of us (DMN) is from NASA Grant NCCI-42.

\section{REFERENCES}

Adams, J. H., Jr.; Silberberg, R.; and Tsao, C. H.; 1981: Cosmic Ray Effects on Microelectronics. Part I-The Near-Earth particle Environment. NRL Memo. Rep. 4506-Pt. I, U.S. Navy, Aug. 1981. (Available from DTIC as AD A103 897).

Barkas, W. H.; 1963: Nuclear Research Emulsions, vol. 1, Academic Press, New York.

Blakely, E. A.; Tobias, C. A.; Yang, T. C. H.; Smith, K. C.; and Lyman, J. T.; 1979: Inactivation of human kidney cells by high energy monoenergetic heavy ion beams. Radiat. Res. 80:122-160.

Bertini, H. W.; 1970: MECC7: a Monte Carlo Intranuclear Cascade and Evaporation Code. Oak Ridge, Tennessee: Oak Ridge National Laboratory, Radiation Shielding Information Center.

Butts, J. J.; and Katz, R.; 1967: "Theory of RBE for Heavy Ion Bombardment of Dry Enzymes and Viruses.," Radiat. Res. 30:855-871.

Cucinotta, F. A.; Atwell, W.; Hardy, A. C.; Golightly, M. J.; Wilson, J. W.; Townsend, L. W.; Shinn, J.; Nealy, J. E.; and Katz, R.; 1990: "Predications of Cell Damage Rates for Lifesat Missions." NASA TM 102170.

Cucinotta, F. A.; Hajnal, F.; and Wilson, J. W.; 1990: Energy deposition at the bonetissue interface from target fragments produced by high energy nucleons. Health Phys. 59:819-825.

Cucinotta, F. A.; Katz, R.; Wilson, J. W.; Townsend, L. W.; Nealy, J. E.; and Shinn, J. L.; 1991a: "Cellular Track Model of Biological Damage to Mammalian Cell Cultures from Galactic Cosmic Rays." NASA TP 3055.

Cucinotta, F. A.; Atwell, W.; Weyland, M.; Hardy, A. C.; Wilson, J. W.; Townsend, L. W.; Shinn, J. L.; and Katz, R.; 1991b: "Radiation Risk Predictions for Space Station Freedom Orbits." NASA TP 3098.

Cucinotta, F. A.; Katz, R.; Wilson, J. W.; Townsend, L. W.; Shinn, J. L.; and Hajnal, F.; 1991c: Biological Effectiveness of High Energy Protons: Target Fragmentation. Radiat. Res. 127. 
Dertinger H.; and Jung, H.; 1970: Molecular Radiation Biology, Springer-Verlag, New York.

Curtis, S. B.; Townsend, L. W.; and Wilson, J. W.; 1990.

Goodhead, D. T.; 1988: "Spatial and Temporal Distribution of Energy," Health Physics 55:231-240.

Goodhead, D. T.; 1989: "Relationships of radiation track structure to biological effect: a re-interpretation of the parameters of the Katz model," Nuclear Tracks and Radiation Measurements 16, 177-184.

Greiner, D. E., et al.; 1975: Momentum distributions of isotopes produced by relativistic 12-C and 16-O projectiles. Phys. Rev. Lett. 35:152-155.

Hall, E. J.; Kellerer, A. M.; Rossi, H. H.; and Lam, Y. P.; 1978: The relative biological effectiveness of $160 \mathrm{MeV}$ protons-II. Int. J. Radiat. Onc. Bio. Phys. 4, 1009-1013.

ICRP; 1977: Recommendations of the International Commission on Radiological Protection. ICRP Publ. 26, Pergamon Press, Jan. 17, 1977.

Janni, J. F.; 1982: "Proton Range-Energy Tables, 1 keV-10 GeV," At. Data and Nucl. Data Tables 27:147-529.

Katz, R.; 1978a: "Track Structure Theory in Radiobiology and in Radiation Detection," Nucl. Track Detection 2:1-28.

Katz, R.; 1978b: "High LET Constraints on Low LET Survival," Phys. Med. Biol. 23:909-916.

Katz, R.; 1984: "Formation of Etchable Tracks in Plastics," Nuclear Tracks and Radiation Measurements 8:1-4.

Katz, Robert: Biological Effects of heavy Ions From the Standpoint of Target Theory. Adv. Space Res., vol. 6, no. 11, 1986, pp. 191-198.

Katz, R.; 1990a: "Cross Section," Appl. Radiat. Isot. 41:563-567.

Katz, R.; 1990b: "On the Normalized Yield (events/rad/dalton) of Biological Molecules Irradiated with Energic Heavy Ions," Radiation Physics and Chemistry in press.

Katz, R; Ackerson, R.; Homayoonfar, M.; and Sharma, S. C.; 1971: Inactivation of cells by heavy ion bombardment. Radiat. Res. 47:402-425.

Katz, R.; and Cucinotta, F. A.; 1991: "RBE vs. Dose for Low Doses of High LET Radiations," Health Physics 5:717-718.

Katz, R.; Dunn, D. E.; and Sinclair, G. L.; 1985: "Thindown in Radiobiology," Radiat. Prot. Dosimetry 13:281-284.

Katz, R.; and Huang, GuoRong; 1989: "Track Core Effects in Heavy Ion Radiolysis," Physics and Chemistry 33:345-349.

Katz, R.; Haung, G.; 1991: "Radiosensitivity Parameters for Cell Survival in Tradescantia and for Chromosome Aberrations in Chinese Hamster Cells," Radiation Protection Dosimetry 31:372-374.

Katz, R.; Loh, Kim Sum; Daling, Luo; and Huang, GuoRong; 1990: "An Analytic Representation of the Radial Distribution of Dose from Energetic Heavy Ions in Water, Si, $\mathrm{LiF}, \mathrm{NaI}$, and $\mathrm{SiO}_{2}$ " Radiation Effects and Defects in Solids 114:15-20.

Katz, R.; and Kobetich, E. J.; 1968: "Response of NaI(T1) to Energetic Heavy Ions," Phys. Rev. 170:397-400.

Katz, R.: and Kobetich, E. J.; 1969: "Particle Tracks in Emulsion," Phys. Rev. 186:344-351.

Katz, R.; Sharma, S. C.; and Homayoonfar, M.; 1972: "The Structure of Particle Tracks," in Topics in Radiation Dosimetry, ed. F. A. Attix, Academic Press, New York.

Katz, R.; Sinclair, G. L.; and Waligorski, M. P. R.; 1986: "The Fricke Dosimeter as a 1-hit Detector," Nucl. Tracks Radiat. Meas. 11:301-307. 
Katz, R.; and Wesely, S.; 1991: "Cross Sections for Single and Double Strand Breaks in SV-40 Virus in $E_{0}$ Buffer after Heavy Ion Irradiation. Experiment and Theory," Radiation and Environmental Biophysics 30:81-85.

Katz, R.; and Varma, M. N.; 1990: "Radial Distribution of Dose," to be published.

Katz, R.; and Zachariah, R.; 1991: E. Coli B modeled as a 1-hit detector. In: Radiation Research, a Twentieth Century Perspective, J. D. Chapman, W. C. Dewey, and G. F. Whitmore, eds. Acadamic Press, San Diego, p. 117.

Kellerer, A. M.; and Brenot, J.; 1973: Nonparametric determination of modifying factors in radiation action. Radiat. Res. 56:28-29.

Silberberg, R.; Tsao, C. H.; and Shapiro, M. M.; 1976: Semiempirical Cross Sections. In: Spallation Nuclear Reactions and Their Applications (B. S. P. Shen and M. Merker, Eds.) pp. 49-81. Dardrecht Publishing Co., Boston, MA.

Skarsgard, L. D.; Kihlman, B. A.; Parker, L.; Pujara, C. M.; and Richardson, S.; 1967: Survival chromosome abnormalities, and recovery in heavy-ion and X-irradiated mammalian cells. Radiat. Res. Suppl. 7:208-221.

Todd, P.; 1967: Heavy ion inactivities of cultured human cells. Radiat. Res. Suppl. 7:196-207.

Townsend, Lawrence W.; Nealy, John E.; Wilson, John W.; and Simonsen, Lisa C.; 1990: Estimates of Galactic Cosmic Ray Shielding Requirements During Solar Minimum. NASA TM-4167.

Underbrink, A. G.; Huczkowski, J.; Woch, B; Gedlek, E.; Cebulska-Wasilweska, A.; Litwiniszyn, M.; and Kasper, E.; 1978: "The Relationship of Different Somatic Mutations Induced by Neutrons and X Rays to Loss of Reproductive Integrity in Tradescantia Stamen Hairs," Report 1039/B, 1978, Institute of Nuclear Physics, Krakow (D).

Waligorski, M. P. R.; Hamm, R. N.; and Katz, R.; 1986: "The Radial Distribution of Dose Around the Path of a Heavy Ion in Liquid Water," Nucl. Tracks Radiat. Meas. 11:309-319.

Waligorski, M. P. R.; Loh, K. S.; and Katz, R.; 1987: "Inactivation of Dry Enzymes and Viruses by Energetic Heavy Ions," Radiat. Phys. Chem. 30:201-208.

Waligorski, M. P. R.; Danialy, G.; Loh,K. S.; and Katz, R.; 1989: "Response of the alanine dosimeter to charge particle and neutron irradiations," Applied Radiation and Isotopes 40:923-933.

Waligorski, M. P. R.; Sinclair, G. L.; and Katz, R.; 1987: "Radiosensitivity Parameters for Neoplastic Transformation in C3H10T1/2 Cells," Radiat. Res. 111:424-437.

Wilson, J. W.; 1777a: "Analysis of the Theory of High Energy Transport." NASA TN D-8381.

Wilson, J. W.; 1977b: Depth dose relations for heavy ion beams. Va. J. of Sci., vol. 28:136-138.

Wilson, J. W.; 1983: "Heavy Ion Transport in the Straight Ahead Approximation." NASA TP-2178.

Wilson, J. W., et al. 1989: BRYNTRN: A Baryon Transport Computer Code. NASA TM-4037 (1988), also NASA TP-2887 (1989).

Wilson, J. W.; Lamkin, S. L.; 1976: Perturbation theory for charged-particle transport in one dimension. Nucl. Sci. \& Eng. 57:292-299.

Wilson, J. W.; Townsend, L. W.; and Khan, F.; 1989: Evaluation of highly ionizing components in high-energy nucleon radiation fields. Health Phys. 57:717-724.

Wilson, J. W.; Townsend, L. W.; and Badavai, F. F.; 1984: Galactic HZE propagation through the earth's atmosphere. Radiat. Res. 109:173-183.

Yang, T. C.; Craise, L. M.; and Tobias, C. A.; 1985: "Neoplastic Cell Transformation by Heavy Charged Particles," Radiat. Res. Suppl. 104:S177-S187.

Ziegler, J. F.; 1980: Stopping Cross-Sections for Energetic Ions in All Elements. Pergamon Press, New York, 1980. 